\title{
Non-Geostrophic and Non-Hydrostatic Stability of a Baroclinic Fluid*
}

\author{
By Tatsushi Tokioka \\ Geophysical Institute, Tokyo University, Tokyo \\ (Manuscript received 8 May 1970, in revised form 5 September 1970)
}

\begin{abstract}
Non-geostrophic and non-hydrostatic effects on the stability of Eady's baroclinic model are studied in the range of small Richardson numbers $(R i)$. The latitudinal as well as the longitudinal variations of wave perturbations are included. There are two growth rate maxima, one is that of the ordinary baroclinic instability on the $l$ (latitudinal wave number) $=0$ axis, and the other is that of a symmetric instability on the $k$ (longitudinal wave number) $=0$ axis and $|l| \rightarrow \infty$ when $R i<1$. There appears an unstable mode destabilized by the inertial effect. The non-hydrostatic effect supresses the appearance of Helmholtz type instability in the range of positive $R i$. There is little difference between eigenvalues in the case $(k, l)$ and $(k,-l)$, but the structure of perturbations in the two cases differs greatly with the increase of $|l|$. The effect of the $\beta$-term is also studied under non-geostrophic and non-hydrostatic conditions.
\end{abstract}

\section{Introduction}

The stability problem of a baroclinic zonal current has been studied extensively by many authors, notably Charney (1947) and Eady (1949). Their results had a remarkable success in explaining the mechanisms of the development of long waves in the middle latitudes. They used geostrophic and hydrostatic approximations, which are appropriate for scales of long waves in the middle latitudes. Eady's model does not include the latitudinal variation of the Coriolis parameter (the $\beta$-term) and has a rigid upper lid. Owing to these simplifications, Eady's model becomes symmetric with respect to the mid-level and allows analytical solutions for both unstable baroclinic waves and neutral waves. This is one of the exceptional cases in hydrodynamic stability problems (see Bretherton (1966)). In Eady's model, there is a short wave cutting of baroclinic instability and the most preferred scale does not depend on the vertical wind shear. On the other hand, Charney's model includes the effect of the $\beta$-term and there is no short wave cutting. The existence of higher modes of baroclinic waves in Charney's model was later clarified by Green (1960) and Burger (1962).

\footnotetext{
* Division of Meteorology, Contribution No. 190
}

Several authors have examined non-geostrophic effects on the baroclinic stability problem from various motivations. Fjørtoft (1950) studied a special case of neutral static stability and obtained a finite short wave cutting. 'Arnason (1963) investigated non-geostrophic effects in an attempt to apply the stability problem to the low latitudes, where the geostrophic balance is considered to be poorly attained. He showed that the largest Rossby number for which baroclinic unstable waves can exist (the critical Rossby number) becomes small owing to the non-geostrophic effect.

Stone (1966) investigated this problem with the application to the atmospheres of the major planets of the solar system in mind. He included sinusoidal variation of perturbations in the latitudinal direction and studied several limiting cases. They are:
(i) $k$ (longitudinal wave number) $\rightarrow 0$,
(ii) $l$ (latitudinal wave number) $\rightarrow 0$, for small $k$,
(iii) $l \rightarrow 0, k \rightarrow \infty$.

In case (i) a symmetric instability appears when the Richardson number $(R i)$ is less than 1 . The instability attains its maximum growth rate at $k=0$ and $l \rightarrow \pm \infty$. In case (ii) the ordinary baroclinic instability appears for a region of small Rossby numbers. In case (iii) Helmholtz type instability appears when $R i$ becomes less than $1 / 4$. Stone (1966) further discussed the position of the 
maximum growth rate among the limiting cases. $\mathrm{He}$ did not, however, discuss instabilities in other part of the wave number space. He also left the study of the structure and the energetics of unstable waves.

Recently Gambo (1970b) studied a baroclinic stability property at a small Rossby number and suggested a possibility of growth rate maximum at a moderate $l$ and a small $k$ when $R i$ is less than 1. But he used geostrophic and hydrostatic approximations in his discussions.

In the present paper, we investigate the nongeostrophic and non-hydrostatic effects from the following motivations. One is to inquire the possibility to find a medium scale unstable wave in the baroclinic model at small $R i$. It is observationally known that in a frontal zone such as the "Baiu" front, a persistent phenomenon in the lower atmosphere near Japan or over China during the season of June or July, small scale cyclones often travel eastwards along the front. In such a situation, $R i$ is thought to be small (of order unity) owing to a large wind shear and a small static stability. In other words, we inquire to what extent we can explain such medium scale disturbances by a simple baroclinic model which does not include the effect of cumulus convection explicitly. Another motivation is to study the structure and the energetics of the various unstable waves precisely, which have not yet been made. It is also interesting to study inertial and nonhydrostatic effects on the stability in the range of wavelengths shorter than the critical wavelength of the Eady's mode.

In the last part of this paper, the effect of the $\beta$-term is also studied under non-geostrophic and non-hydrostatic conditions. 'Arnason (1963) partly studied this problem and concluded the reappearance of the short wave cutting of the ordinary baroclinic mode, contrary to the conclusion under geostrophic assumptions (see Green (1960) or Burger (1962)).

\section{The fluid model}

Several baroclinic models have been presented so far. They are classified according to the upper boundary condition and inclusion or exclusion of the $\beta$-effect. Eady (1949) used a Bousssinesq fluid model, which has a rigid upper boundary and neglects the $\beta$-effect. This model is simple both physically and mathematically but expresses the essential feature of baroclinic fluids. In the fol- lowing we extend Eady's theory to non-geostrophic and non-hydrostatic cases with inclusion of the $\beta$-effect.

\subsection{Governing equations}

We use a Boussinesq system of equations. Equations of motion, thermodynamic equation and continuity equation become as follows:

$$
\begin{aligned}
& \frac{d u}{d t}-f v=-\frac{\partial p}{\partial x}, \\
& \frac{d v}{d t}+f u=-\frac{\partial p}{\partial y}, \\
& \frac{d w}{d t}-g \alpha \theta=-\frac{\partial p}{\partial z}, \\
& \frac{d \theta}{d t}=0, \\
& \frac{\partial u}{\partial x}+\frac{\partial v}{\partial y}+\frac{\partial w}{\partial z}=0,
\end{aligned}
$$

where $\frac{d}{d t}=\frac{\partial}{\partial t}+u \frac{\partial}{\partial x}+v \frac{\partial}{\partial y}+w \frac{\partial}{\partial z} . \quad u, v$ and $w$ are velocity components in $x$-(eastward), $y$-(northward) and $z$-(upward) directions respectively. $p$ is the deviation from hydrostatic pressure divided by a mean density and $\theta$ the temperature deviation from a reference temperature corresponding to the mean density. $g$ is the accerelation of gravity, $\alpha$ the coefficient of thermal expansion and $f$ the Coriolis factor.

We assume a basic flow $U$ in the $x$-direction, which varies linearly with $z$ in the range $-z_{0} / 2$ $\leq z \leq z_{0} / 2$ :

$$
U=\Lambda z, \quad \Lambda: \text { positive constant. }
$$

We choose a temperature field as

$$
\theta=B y+S z, \quad B, S: \text { constats. }
$$

Inserting (2-6) and (2-7) into Eqs. (2-2) and (2-3), we get the thermal wind relation

$$
f \Lambda=-g \alpha B \text {. }
$$

Equations governing small perturbations superimposed upon the basic state are written as follows:

$$
\begin{aligned}
& i k(U-c) u^{\prime}+A w^{\prime}-f v^{\prime}=-i k p^{\prime}, \\
& i k(U-c) v^{\prime}+f u^{\prime}=-i l p^{\prime}, \\
& i k(U-c) w^{\prime}-g \alpha \theta^{\prime}=-\frac{\partial p^{\prime}}{\partial z}, \\
& i k(U-c) \theta^{\prime}+B v^{\prime}+S w^{\prime}=0, \\
& i k u^{\prime}+i l v^{\prime}+\frac{\partial w^{\prime}}{\partial z}=0
\end{aligned}
$$


where normal mode form $\phi=\phi e^{i(k x+l y-k c t)}$ is assumed to each component of perturbations. (The longitudinal wave number $k$ is limited to be positive or zero in the following discussions.) Primes denote perturbation quantities. Hereafter we omit primes unless otherwise stated. From (2-9) and (2-10), we obtain a vorticity equation

$$
i k(U-c)(i k v-i l u)=f \frac{d w}{d z}+i l \Lambda w-\beta v,(2-14)
$$

where $\beta=\frac{d f}{d y}$. Also from (2-9) and (2-11),

$$
i k(U-c)\left(\frac{d u}{d z}-i k w\right)=f \frac{d v}{d z}+i l \Lambda v-i k g \alpha \theta,
$$

is derived. If we express horizontal components of velocity as

$$
\boldsymbol{V}_{h}=(u, v)=\nabla_{h} \chi+\nabla_{h} \phi \times \boldsymbol{k},
$$

where $\boldsymbol{k}$ denotes a unit vector in $z$-direction, we get from Eq. (2-13)

$$
\nabla h \chi=\nabla h \frac{d w}{d z} / M, \quad M=k^{2}+l^{2} .
$$

Substituting the expression (2-16) into (2-14), and eliminating $\chi$ between (2-14) and (2-17), we obtain the following expression,

$$
\begin{aligned}
\nabla_{h} \psi \times \boldsymbol{k} & =\frac{f-i \frac{l \beta}{M}}{i k[(U-c) M-\beta]} \nabla h \frac{d w}{d z} \times \boldsymbol{k} \\
& +\frac{l \Lambda}{k[(U-c) M-\beta]} \nabla h w \times \boldsymbol{k} \\
& +\frac{\beta}{i k[(U-c) M-\beta]}\left(\frac{d w}{d z}, 0\right) .
\end{aligned}
$$

Also after elimination of $\theta$ between (2-12) and (2-15), we obtain

$$
\begin{aligned}
& k\left[\frac{g \alpha S}{k(U-c)}-k(U-c)\right] w-i k(U-c) \frac{d u}{d z} \\
& \quad+\Lambda\left(i l-\frac{f}{U-c}\right) v+f \frac{d v}{d z}=0 .
\end{aligned}
$$

Substituting the expressions (2-17) and (2-18) into (2-19), we finally get the governing equation for $w$ as,

$$
\begin{gathered}
k(U-c)\left[k(U-c)\left\{k(U-c)-2 \frac{\beta}{k}\right\}-f^{2}\right] \frac{d^{2} w}{d z^{2}} \\
+\Lambda\{[f k(2 M(U-c)-\beta)+i k l \beta(U-c)] \\
\times\left[\frac{f-i \frac{l \beta}{M}}{M(U-c)-\beta}-i \frac{l}{M}\right]
\end{gathered}
$$

$$
\begin{aligned}
& \left.+\frac{M \beta k(U-c)^{2}}{M(U-c)-\beta}\right\} \frac{d w}{d z}+k[M(U-c)-\beta] \\
& \times\left[g \alpha S-k^{2}(U-c)^{2}-\frac{l \Lambda^{2}}{[M(U-c)-\beta]^{2}}\right. \\
& \times\{l \beta(U-c)-2 i f M(U-c)+i f \beta\}] w=0,
\end{aligned}
$$

or with the introduction of a new independent variable $Y=k(U-c) / f$,

$$
\begin{aligned}
Y & {[Y(Y-2 a)-1] \frac{d^{2} w}{d Y^{2}}+\left\{\left[F+\left(\frac{M}{k}\right.\right.\right.} \\
& \left.+i l a) Y]\left[\frac{1-i \frac{l k a}{M}}{F}-i \frac{l}{M}\right]+\frac{\frac{a M}{k} Y^{2}}{F}\right\} \frac{d w}{d Y} \\
& +\frac{f^{2} F}{k \Lambda^{2}}\left\{\frac{g \alpha S}{f^{2}}-Y^{2}-\frac{l A^{2}}{f^{2} F^{2}}[l a Y-i(F\right. \\
& \left.\left.\left.+\frac{M}{k} Y\right)\right]\right\} w=0,
\end{aligned}
$$

where the following abbreviations are used:

$$
\begin{aligned}
& a=\beta / f k, \\
& F=\frac{M}{k} Y-k a .
\end{aligned}
$$

Boundary conditions are $w=0$ at $z= \pm z_{0} / 2$ or at $Y=Y\left( \pm z_{0} / 2\right)$. If we set $\beta$ to zero, Eq. $(2-20)^{\prime}$ becomes

$$
\begin{aligned}
& Y\left(Y^{2}-1\right) \frac{d^{2} w}{d Y^{2}}+2\left(1-i \frac{l}{k} Y\right) \frac{d w}{d y} \\
& +\frac{M}{k^{2}} Y\left[R i-\frac{f^{2} Y^{2}}{\Lambda^{2}}+2 i \frac{k l}{M Y}\right] w=0,
\end{aligned}
$$

where $R i=g_{\alpha} S / \Lambda^{2}$.

It is noteworthy that if $Y$ and $l$ are replaced by $-Y$ and $-l,(2-20)^{\prime \prime}$ has a solution $w(-Y)$ where $w(Y)$ is a solution for $Y$ and $l$, and that if $Y$ and $l$ are replaced by $Y^{*}$ (complex conjugate) and $-l,(2-20)^{\prime \prime}$ also has a solution $w^{*}\left(Y^{*}\right)$. In short Eq. $(2-20)^{\prime \prime}$ is invariant for the change of $Y$ to $-Y^{*}$, i.e., if $c$ is an eigenvalue of $(2-20)^{\prime \prime}$, $-c^{*}$ also becomes an eigenvalue. We remark that under a further restriction $l=0$, Eq. $(2-20)^{\prime \prime}$ becomes very simple and has a high degree of symmetry, i.e., if $c$ is an eigenvalue, $-c$ and $\pm c^{*}$ are also eigenvalues. The asymmetry in the case of nonvanishing $l$ implies that the growth rate differs between the cases $(k, l)$ and $(k,-l)$.

\subsection{Energy equation}

In the baroclinic model under consideration, perturbation kinetic energy averaged over one 
wavelength $E_{k}$ obtains its energy through the conversion from potential energy $\varepsilon p$, and through the conversion from basic kinetic energy $\varepsilon_{k}$. The averaged energy equation is given by

$$
\frac{d E_{k}}{d t}=\varepsilon_{p}+\varepsilon_{k}-\frac{d}{d z}(\overline{p w}) .
$$

$-\frac{d}{d z}(\overline{p w})$ vanishes when it is further integrated vertically. From Eqs. (2-9) to (2-13), $\varepsilon_{p}$ and $\varepsilon_{k}$ are written in terms of $w$ as follows;

$$
\begin{aligned}
& \varepsilon p=g \alpha \overline{\theta w}=\frac{f A}{2 k M|U-c|^{2}}\left[l\left(U-c_{r}\right)\right. \\
&+\frac{\beta l\left\{\left(U-c_{r}\right)\left(U-c_{r}-\beta / M\right)-c_{i}^{2}\right\}}{M|U-c-\beta / M|^{2}} \\
&\left.-\frac{2 f c_{i}\left(U-c_{r}-\beta / 2 M\right)}{|U-c-\beta / M|^{2}}\right] \frac{d W^{2}}{d z} \\
&-\frac{1}{k|U-c|^{2}}\left[S c_{i}\right. \\
&\left.+\frac{f \Lambda^{2} l\left\{\left(U-c_{r}\right)\left(U-c_{r}-\beta / M\right)-c_{i}^{2}\right\}}{M|U-c-\beta / M|^{2}}\right] W^{2} \\
&-\frac{f \Lambda}{k M|U-c|^{2}}\left[l c_{i}\right. \\
&+\frac{f\left\{\left(U-c_{r}\right)\left(U-c_{r}-\beta / M\right)-c_{i}^{2}\right\}}{|U-c-\beta / M|^{2}} \\
&\left.+\frac{2 \beta l c_{i}\left(U-c_{r}-\beta / 2 M\right)}{M|U-c-\beta / M|^{2}}\right] W^{2} \frac{d \phi}{d z}, \\
& \varepsilon_{k}=-A \overline{u w}=\frac{l^{2} \Lambda^{2} c_{i}}{k M|U-c-\beta / M|^{2}} W^{2}+\frac{\Lambda}{M}[k \\
&\left.+\frac{l\left\{f c_{i}-\beta l\left(U-c_{r}-\beta / M\right) / M\right\}}{k|U-c-\beta / M|^{2}}\right] W^{2} \frac{d \phi}{d z} \\
&+\frac{A l}{2 k M|U-c-\beta / M|^{2}}\left[f\left(U-c_{r}-\beta / M\right)\right. \\
&\left.+\beta l c_{i} / M\right] \frac{d W^{2}}{d z},
\end{aligned}
$$

where $w$ is expressed as $W e^{i \phi}$ ( $\phi$ is a real function and $W$ is a positive and real function of $z$ ). If we set $l$ to zero, $\varepsilon_{k}$ becomes $\frac{\Lambda k}{M} W^{2} \frac{d \phi}{d z}$. Then it is clear that when $l=0$, the phase line of $w$ must incline westward to make $\varepsilon_{k}$ positive even in the non-geostrophic case.

\section{Computational procedure}

The problem is now to solve Eq. $(2-20)$ under the boundary conditions $w=0$ at $z= \pm z_{0} / 2$. When we solve Eqs. (2-9) to (2-13) instead of (2-20), matrix method is usually used. Matrix method has a merit that all the eigenvalues and eigen- functions are obtained at once but at the same time has a disadvantage that the rank of the matrix must be increased as $3 n-2$, where $n$ is the number of subdivisions in $z$-direction. In the following, we solve Eq. (2-20) by an iterative method as was used by Orlanski (1968). The method is powerful when we wish to obtain an eigenvalue and the corresponding eigenfunction accurately with a small machine time.

We can safely assume that $w$ is differenciable with respect to $c$. The governing equation for $\frac{d w}{d c}$ is obtained from Eq. (2-20) as;

$$
\begin{aligned}
& k(U-c)[k(U-c)\{k(U-c)-2 \beta / k\} \\
& \left.-f^{2}\right] \frac{d^{2}}{d z^{2}}\left(\frac{d w}{d c}\right)+\Lambda\{[f k(2 M(U-c)-\beta) \\
& +i k l \beta(U-c)] \times\left[\frac{f-i \frac{l \beta}{M}}{M(U-c)-\beta}-i \frac{l}{M}\right] \\
& \left.+\frac{M \beta k(U-c)^{2}}{M(U-c)-\beta}\right\} \frac{d}{d z}\left(\frac{d w}{d c}\right) \\
& +k[M(U-c)-\beta] \times\left[g \alpha S-k^{2}(U-c)^{2}\right. \\
& -\frac{l A^{2}}{[M(U-c)-\beta]^{2}}\{l \beta(U-c) \\
& -2 i f M(U-c)+i f \beta\}] w=G(w, z),
\end{aligned}
$$

where

$$
\begin{aligned}
G(w, z) & =k\left[3 k^{2}(U-c)^{2}-4 \beta(U-c)\right. \\
& \left.-2 f^{2}\right] \frac{d^{2} w}{d z^{2}}+\Lambda\{[2 M f \\
& +i l \beta] \times\left[\frac{f-i \frac{l \beta}{M}}{M(U-c)-\beta}-i \frac{l}{M}\right] \\
& -\frac{M\left(f-i \frac{l \beta}{M}\right)}{[M(U-c)-\beta]^{2}} \\
& \times[f(2 M(U-c)-\beta)+i l \beta(U-c)] \\
& \left.+\frac{M \beta(U-c)[M(U-c)-2 \beta]}{[M(U-c)-\beta]^{2}}\right\} \frac{d w}{d z} \\
& +k M\left\{g \alpha S-3 k^{2}(U-c)^{2}\right. \\
& +2 \frac{k^{2}}{M} \beta(U-c)+\frac{l A^{2}}{[M(U-c)-\beta]^{2}} \\
& \times[l \beta(U-c)-2 i f M(U-c)+i f \beta] \\
& \left.+\frac{l A^{2}}{M(U-c)-\beta}\left(2 i f-\frac{l \beta}{M}\right)\right\} w .
\end{aligned}
$$

As the value of $w$ at $z=-z_{0} / 2$ is zero, the boundary condition for Eq. (3-1) becomes $d w / d c$ 
$=0$ there. Thus we set $w=\frac{d w}{d c}=0$ at $z=-z_{0} / 2$. Further we can specify $\frac{d w}{d z}$ artificially at $z=$ $-z_{0} / 2$, independent of $c$. Then $\frac{d}{d z}\left(\frac{d w}{d c}\right)=0$ is obtained. For a certain guess for $c$, integration of $(2-20)$ and (3-1) is performed with respect to $z$ under the above boundary conditions. At $z=$ $z_{0} / 2, w$ does not usually satisfy the boundary condition. The correction of eigenvalue $\Delta c$ is then estimated as follows;

or

$$
\left.w\right|_{z_{0} / 2}+\left.\frac{d w}{d c}\right|_{z_{0} / 2} \cdot \Delta c=0
$$

$$
\Delta c=-\left.w\right|_{z_{0} / 2} /\left.\frac{d w}{d c}\right|_{z_{0} / 2}
$$

This process is repeated until the accuracy desired is attained. For most of the calculations, the fluid layer was divided into 30 intervals, which gave us enough accuracy.

\section{Comments on several instabilities}

Before discussing the complete solutions of (220 ), we shall review several instabilities presented so far, which are obtained under some restrictions on $(2-20)$ or $(2-20)^{\prime \prime}$.

\subsection{Eady's baroclinic instability $(l=0)$}

Eady (1949) treated Eqs. (2-9) to (2-13) under geostrophic and hydrostatic assumptions. When $l=0,(2-20)^{\prime \prime}$ becomes

$$
\frac{d^{2} w}{d Y^{2}}-\frac{2}{Y} \frac{d w}{d Y}+R i w=0
$$

under those assumptions. We also obtain an equation for $p$,

$$
(U-c)\left(\frac{d^{2} p}{d z^{2}}-\frac{g \alpha S k^{2}}{f^{2}} p\right)=0,
$$

under the same assumptions. The boundary conditions for Eq. (4-2) are $(U-c) \frac{d p}{d z}-\Lambda p=0$ at $z= \pm z_{0} / 2$. Clearly Eq. (4-1) with the boundary conditions $w=0$ at $z= \pm z_{0} / 2$ is equivalent to Eq. (4-2) with the boundary conditions $(U-c) \frac{d p}{d z}$ $-\Lambda p=0$ at $z= \pm z_{0} / 2$ as an eigenvalue problem.

As Eady (1949) showed, the level where $U-c$ $=0(Y=0)$ is only an "apparent" singular point in (4-1), which is clearly seen from Eq. (4-2). The solutions of Eq. (4-2) are easily obtained.
They are expressed in terms of $R_{o} \equiv k \Lambda z_{0} / 2 f$ (the Rossby number) and $R i$ as follows;

(i) unstable mode

$$
\begin{aligned}
p & =\left[(1+\delta)^{2}+\alpha^{2}\right] e^{\delta(\xi-1)}+\left[\delta^{2}+\alpha^{2}-1\right. \\
& +2 i \alpha] e^{-\delta(\xi-1)},
\end{aligned}
$$

where

$$
\begin{aligned}
& \delta=R i^{1 / 2} R_{o}, \quad \xi=2 z / z_{0}, \quad \alpha= \pm k c i R i, \\
& \alpha^{2}=2 \delta \operatorname{coth} 2 \delta-\delta^{2}-1(>0), \quad C_{r}=U(0)
\end{aligned}
$$

(ii) stable mode

$$
\begin{aligned}
& p^{+}=(\delta-\gamma+1) e^{\delta \xi}+(\delta-\gamma-1) e^{-\delta \xi}, \\
& c^{+}=U(0)+\gamma / k R i, \\
& p^{-}=(\delta+\gamma+1) e^{\delta \xi}+(\delta+\gamma-1) e^{-\delta \xi}, \\
& c^{-}=U(0)-\gamma / k R i,
\end{aligned}
$$

where

$$
\gamma=\left(1+\delta^{2}-2 \delta \operatorname{coth} 2 \delta\right)^{1 / 2} .
$$

The short wave cutting of the instability is expressed as;

$$
1+\delta^{2}=2 \delta \operatorname{coth} 2 \delta
$$

or

$$
\delta=R i^{1 / 2} R_{o}=1.199 \cdots .
$$

The most preferred mode is also expressed as

$$
R i^{1 / 2} R_{o}=0.8031 \cdots .
$$

We remark that the unstable solutions are completely expressed in terms of two parameters, for example $R_{o}$ and $R i$, under the hydrostatic approximation. This will be contrasted to the nonhydrostatic case where three parameters are needed.

\subsection{Baroclinic instability when $l \neq 0$}

When we include the latitudinal variation of perturbations $(l \neq 0)$, Eq. $(2-20)^{\prime \prime}$ is transformed into

$$
\begin{aligned}
& \frac{d^{2} R}{d Y^{2}}-\frac{2}{Y} \frac{d R}{d Y}-n^{2} R=0, \\
& n^{2}=R i-\left(\frac{l}{k}\right)^{2}(1-R i),
\end{aligned}
$$

under geostrophic and hydrostatic assumptions, where $R$ is related to $w$ as:

$$
w=\left(\frac{1-Y}{1+Y}\right)^{i l / 2 k} R \text {. }
$$

Eady (1949) solved Eq. (4-7) with further approximation $R i \gg 1$, i.e., $n^{2}=R i\left(1+\frac{l^{2}}{k^{2}}\right)$.

Recently Gambo (1970b) treated the case $n^{2}=$ 
$R i-\left(\frac{l}{k}\right)^{2}(1-R i)$. In the latter case $n^{2}$ can be negative when $R i$ is less than 1 and as a consequence the behaviour of the soluitons and eigenvalues becomes different from those in the case of positive $n^{2}$. When $n^{2}$ is negative or zero, the following characteristic equations and eigensolutions are obtained;

(a) $n^{2}<0$

$$
\begin{aligned}
R & =2 \delta \Sigma\left(\frac{z}{z_{0}}+\frac{1}{2}\right) \sin \left\{2 \delta\left(\frac{z}{z_{0}}-\frac{1}{2}\right)\right\} \\
& +i\left[\left(1+2 \delta^{2} \frac{z}{z_{0}}-\Sigma^{2}\right) \sin \left\{2 \delta\left(\frac{z}{z_{0}}-\frac{1}{2}\right)\right\}\right. \\
& \left.-2 \delta\left(\frac{z}{z_{0}}-\frac{1}{2}\right) \cos \left\{2 \delta\left(\frac{z}{z_{0}}-\frac{1}{2}\right)\right\}\right]
\end{aligned}
$$

where

$$
\begin{aligned}
& \Sigma^{2}=(\tan \delta-\delta)(\cot \delta+\delta) \\
& \delta=\frac{k \Lambda z_{0}}{2 f} \sqrt{-n^{2}}=R_{0} \sqrt{-n^{2}} \\
& \Sigma=\frac{i k(U(0)-c)}{f} \sqrt{-n^{2}}
\end{aligned}
$$

(b) $n^{2}=0$

$$
R=\left(\frac{z^{\prime}}{z_{0}}-\frac{1}{2}\right)\left(\frac{z}{z_{0}}+\frac{1}{2}\right)\left(2 \delta \frac{z}{z_{0}}-3 i \Sigma\right)
$$

where

$$
\begin{aligned}
& \delta=R_{o} \\
& \Sigma=\frac{i k}{f}(U(0)-c) \\
& \delta^{2}=3 \Sigma^{2} .
\end{aligned}
$$

The unstable wave region is expressed as follows from the above characteristic equations and also from the results for $n^{2}>0$ including $l$.

i) $R i>1$

$$
\frac{1}{R i-1}\left(\frac{2.39 f}{\Lambda z_{0}}\right)^{2}>l^{2}+\frac{R i}{R i-1} k^{2}
$$

ii) $1>R i>0$

$$
\begin{aligned}
& \frac{1}{1-R i}\left(\frac{\pi f}{\Lambda z_{0}}\right)^{2}>l^{2}-\frac{R i}{1-R i} k^{2}> \\
& -\frac{1}{1-R i}\left(\frac{2.39 f}{\Lambda z_{0}}\right)^{2} .
\end{aligned}
$$

Fig. 1 gives a schematical view of unstable regions in the wave number domain. When $n^{2}<0$, the characteristic equation of (4-8) gives infinite $c_{i}$ (imaginary part of $c$ ) at $\delta=m \frac{\pi}{2}(m= \pm 1, \pm 2 \cdots)$, but as Gambo (1970b) noted, the linear theory may lose its validity in the neighbourhood of
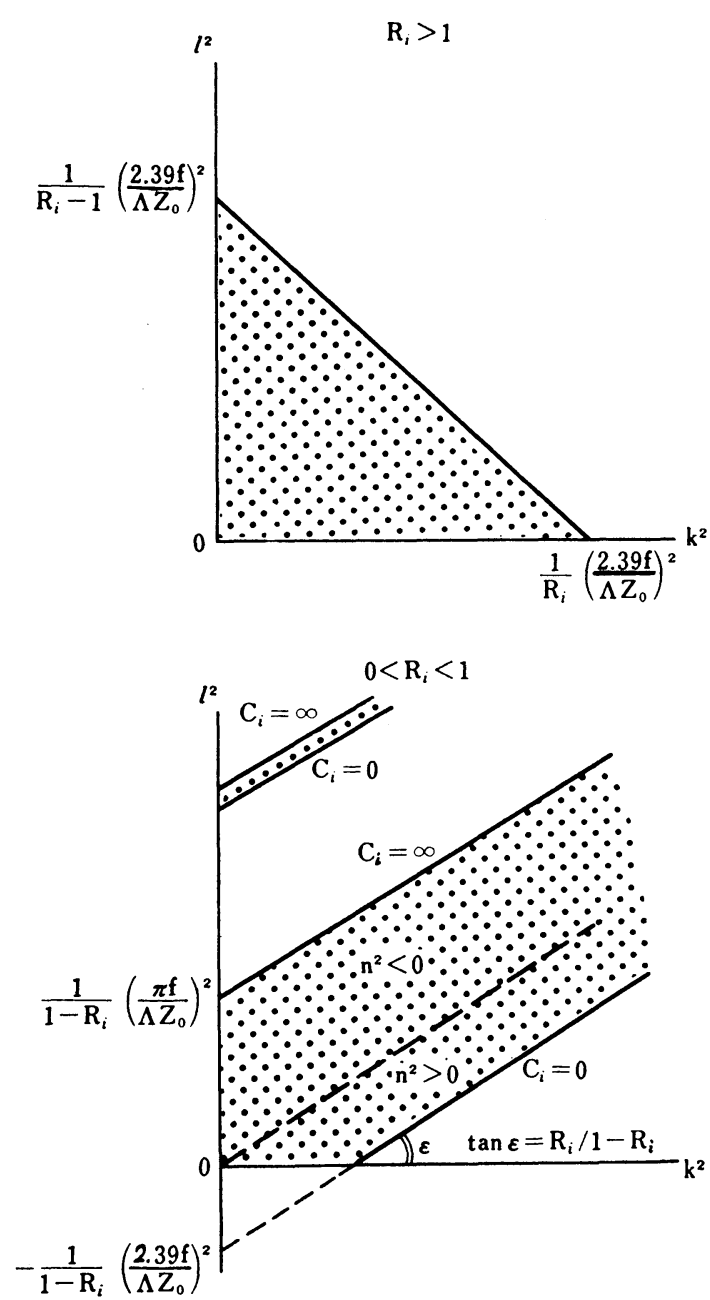

Fig. 1 Unstable domain (strippled area) under geostrophic and hydrostatic assumptions. The ordinate is square of the latitudinal wave number $l$ and the abscissa square of the longitudinal wave number $k . n^{2}$ denotes $R i-(l / k)^{2}(1-R i)$.

infinite growth rates. Also, the adaptation of (4-8) is limited to small $k$ because of the geostrophic assumption. Therefore, it is necessary to treat the problem without the geostrophic assumption to investigate the possibility of a growth rate peak for a moderate value of $l$. Stone (1966) also obtained the characteristic relation corresponding to (4-8) in the limit $k \rightarrow 0$. He showed that near the points $\delta=m \frac{\pi}{2}$, maximum $c_{i}$ becomes finite for this mode by solving an equation under the condition that $k c_{i}$ is finite with inclusion of non-geostrophic effects. 


\subsection{Symmetric instability}

Stone (1966) investigated a limiting case $k \rightarrow 0$ and discussed a symmetric instability and showed the existence of a growth rate maximum on the $k=0$ axis. General theories of symmetric insbility were presented by Fjørtoft (1950) and Ooyama (1966). The limiting case discussed by Stone (1966) corresponds to a special case. Stability tensor (e.g. see Ooyama (1966)) becomes in this case

$$
M=\left(\begin{array}{lr}
f^{2}, & -g_{\alpha B} \\
-g_{\alpha B}, & g_{\alpha} S
\end{array}\right)=\left(\begin{array}{lr}
f^{2} & f \Lambda \\
f \Lambda & g_{\alpha S}
\end{array}\right)
$$

As $\operatorname{Tr} M$ is positive, Det $M<0$ i.e., $R i<1$ is the necessary and sufficient condition for instability. In this case, the maximum growth rate $(=f(1)$ $R i-1)^{1 / 2}$ ) occurs at $l \rightarrow \pm \infty$. If we assume the vertical variation of perturbations as $e^{i m z}$ with neglection of boundary conditions, the most unstable direction, in which disturrbances are apt to elongate, is determined as $\vartheta=\pi-\tan ^{-1}\left(\frac{B}{S}\right)$ (see Ooyama (1966)). $\vartheta$ is the angle measured from the $y$-axis in the meridional plane.

5. Non-geostrophic and non-hydrostatic solutions when $1=\beta=0$

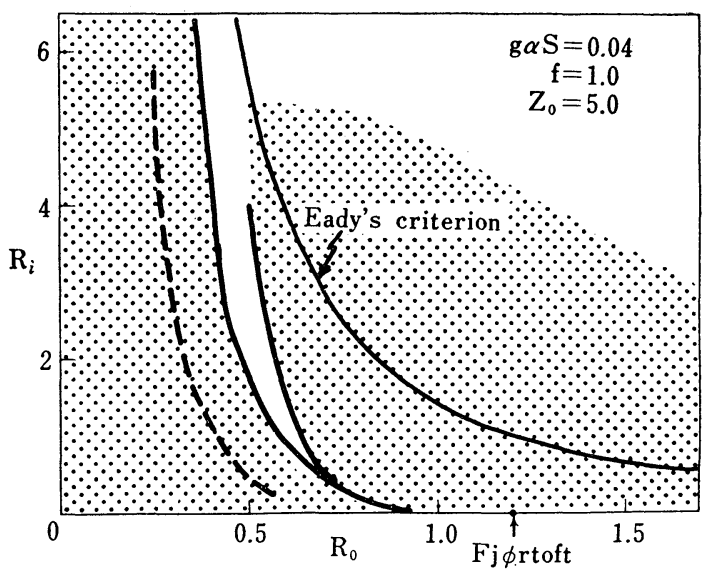

Fig. 2 Stability diagram for non-geostrophic and non-hydrostatic case when $l=\beta=0$. The ordinate is the Richardson number and the abscissa the Rossby number. Unstable domains are strippled. The dashed line denotes the growth rate peak of the baroclinic mode. The thin solid line is Eady's criterion, the left side of which is unstbable under the geostrophic approximation. Fjørtoft's criterion is also indicated.
We solve Eq. (2-20) without assuming geostrophic and hydrostatic balances. Calculations are made with the use of the following parameters: $g_{\alpha} S=0.04 \mathrm{sec}^{-2}, z_{0}=5.0 \mathrm{~cm}$ and $f=1.0 \mathrm{sec}^{-1}$. Fig. 2 shows the region of unstable waves. The ordinate expresses the Richardson number $(R i)$ and the abscissa the Rossby number $\left(R_{o}\right)$. There are two unstable domains, the left of which corresponds to the Eady's mode. The criterion obtained by Eady (1949) under geostrophic and hydrostatic conditions, and the criterion obtained by Fjфrtoft (1950) under non-geostrophic and neutral static stability conditions are also indicated in Fig. 2.

\subsection{Eady's mode}

The critical curve of the Eady's mode moves to smaller $R_{o}$ at a constant $R i$ with the inclusion of non-geostrophic and non-hydrostatic effects. This is consistent with 'Arnason's (1963) result. When we assume the hydrostatic balance, the short wave cutting moves to smaller $R_{o}$ in lesser degree than that under the non-hydrostatic case.

If we formally apply the Eady's criterion to a small $R i$, the most preferred scale doe not change when the static stability is fixed and the vertical shear is changed (see Eq. (4-6)). This is one of the remarkable differences of the geostrophic solution from the non-geostrophic solution obtained above. On the other hand when the vertical shear is fixed and the static stability is decreased, the most preferred scale changes to a smaller wavelength. The maximum growth rate increases with the decrease of Ri. Gambo (1970a) also showed the same results as the criterion of Eady (1949) after appropriate scale considerations.

As stated before, two parameters $R i$ (or $n^{2}$ ) and $R_{o}$ are sufficient to express the unstable mode under the hydrostatic condition, but one more parameter such as $A$ or $g \alpha S$ is necessary in the non-hydrostatic case. Computational results of the growth rate are shown in Fig. 3 with $R i$ as the ordinate and the longitudinal wave number $k$ as the abscissa, where the vertical shear is changed while the static stability $g_{\alpha} S$ is kept constant. As seen in the left side of the figure, the most preferred scale of the Eady's mode changes to larger scale with the decrease of $R i$. The sense of the change of the maximum growth rate and the preferred scale with the change of the static stability at a constant vertical shear agrees with the results of geostrophic models of Eady (1949) and Gambo (1970a). The structure 


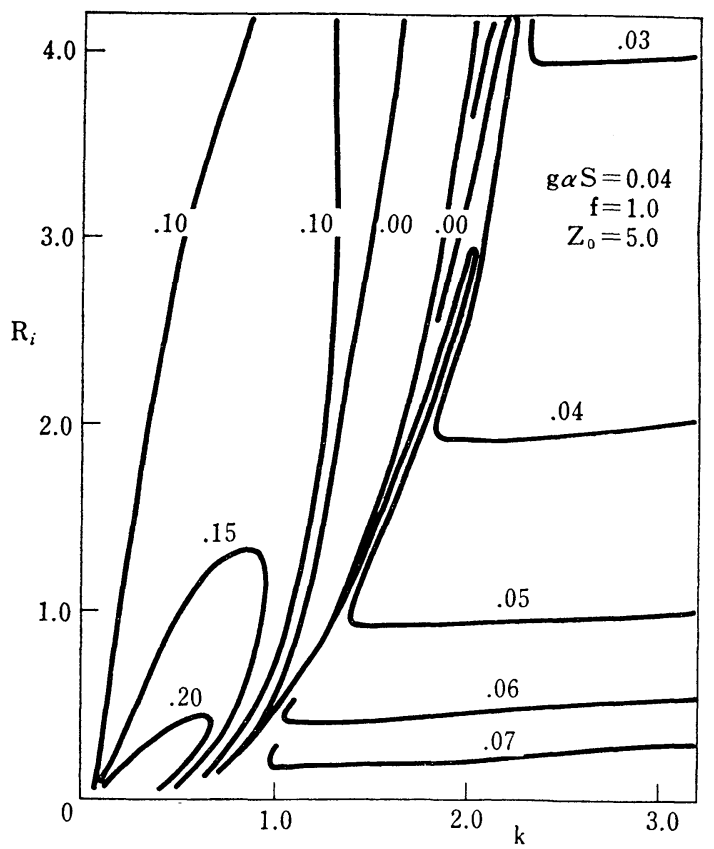

Fig. 3 The growth rate of unstable waves in the case $l=\beta=0$. The abscissa is the longitudinal wave number. (units in c.g.s.)

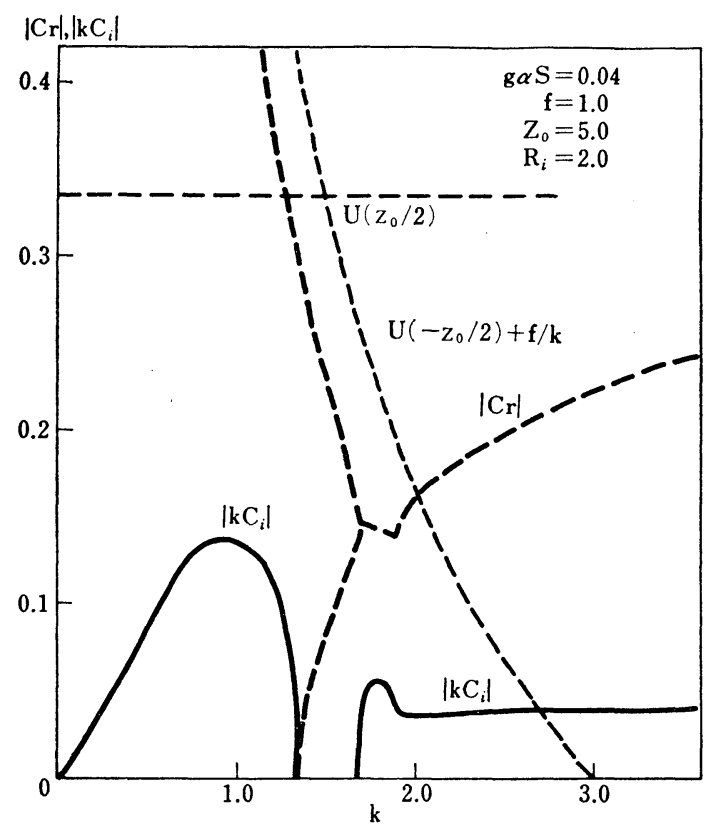

Fig. 4 The growth rate (thick solid line) and the phase velocity (thick dashed line) of unstable waves at $R i=2.0$ when $l=\beta=0$. Two sublines denote $U\left(z_{0} / 2\right)$ and $U\left(-z_{0} /\right.$ 2) $+f / k$. (units in c. g. s.) of the unstable wave at a small $R i$ differes little from that obtained at a large $R i$. As $c_{r}=0$ for this mode, there is a damping wave corresponding to this unstable mode.

\subsection{Another unstable mode}

In the shorter wave range of Figs. 2 and 3, another unstable mode appears after a short range of neutral zone. In the geostrophic case, there appear two neutral waves in the shorter wave range (see Eq. (4-4)). Fig. 4 shows the phase velocity and the growth rate curves obtained at $R i=2$. In the neutral zone, there exist a neutral mode corresponding to $C^{+}$(see eq. (4-4)) and an inertial mode. When the two phase velocities coincide, a new unstable wave appears. Owing to the symmetry of Eq. (2-20) noted before, there are another unstable wave and two damping waves.

In Fig. 4 there are two sublines $U\left(z_{0} / 2\right)$ and $U\left(-z_{0} / 2\right)+f / k$. Below the line $U\left(z_{0} / 2\right)$ a steering level where $U-c_{r}=0$ exists in the range $-z_{0} /$ $2<z<z_{0} / 2$, and on the right side of the curve $U\left(-z_{0} / 2\right)+f / k$ there exists a level where $U-c_{r}$ $+f / k=0$. Bretherton (1966) presented an idea of critical layer instability, i. $e ., c_{i}$ cannot be zero when singular points of the vorticity equation exist in the layers. We shall examine the nature of the neutral modes appeared in the present model. If we set $l$ equal to zero, Eq. $(2-20)^{\prime \prime}$ is written as

$$
Y\left(Y^{2}-1\right) \frac{d^{2} w}{d Y^{2}}+2 \frac{d w}{d Y}+Y\left(R i-\frac{f^{2}}{\Lambda^{2}} Y^{2}\right) w=0
$$

Near the singular point $Y=0$ (or $U-c=0$ ), the above equation becomes

$$
Y \frac{d^{2} w}{d Y^{2}}-2 \frac{d w}{d Y}-Y R i w=0
$$

Eq. $(5-1)^{\prime}$ has two regular solutions

$$
Y^{3 / 2} K_{3 / 2}(\sqrt{R i} Y) \text { and } Y^{3 / 2} I_{3 / 2}\left(\sqrt{R_{i}} Y\right) \text {, }
$$

where $K_{3 / 2}$ and $I_{3 / 2}$ are Bessel functions of imaginary argument of order $3 / 2$. Thus $Y=0$ is still an "apparent" singular point in the non-geostrophic case. Then the appearance of the two neutral waves in Fig. 4 does not contradict the concept of the critical layer instability. Next we examine the singularity at $Y= \pm 1(U-c \pm f / k=0)$. We treat $Y=-1$, for example, then Eq. $(5-1)$ is approximated as

$$
2(Y+1) \frac{d^{2} w}{d Y^{2}}+2 \frac{d w}{d Y}-\left(R i-\frac{f^{2}}{\Lambda^{2}}\right) w=0 .
$$


With the use of new independent variable $\xi=$ $\frac{1}{2}\left(R i-\frac{f^{2}}{\Lambda^{2}}\right)(Y+1)$, the above equation is transformed into

$$
\xi \frac{d^{2} w}{d \xi^{2}}+\frac{d w}{d \xi}-w=0 .
$$

This has a solution of the type $\alpha_{1} K_{0}(2 \sqrt{\xi})+\alpha_{2} I_{0}$ $(2 \sqrt{\xi})$ where $K_{0}$ and $I_{0}$ are Bessel functions of imaginary argument of order 0 . This solution shows that $Y=-1$ is a real singular point, and this suggests that on the right side of the curve $U\left(-z_{0} / 2\right)+f / k$ unstable waves can appear. From the phase velocity curves in Fig. 4, we may infer that the neutral mode $p^{+}$and $p^{-}$are distabilized by the inertial effect. The structure of the unstable wave is shown in Figs. 5a, b, c. Arrows in the figures denote the levels $U-c_{r}=0$ (upper arrow) and $U-c_{r}+f / k=0$ (lower arrow). Near the level $U-c_{r}+f / k=0$, Eqs. (2-21) and (2-22) are approximated as follows

$$
\begin{aligned}
& \varepsilon_{p}=-\frac{A}{k} W^{2} \frac{d \phi}{d z}+\frac{\Lambda c_{i}}{f} \frac{d W^{2}}{d z}-\frac{g \alpha S k c_{i}}{f^{2}} W^{2}, \\
& \varepsilon_{k}=\frac{A}{k} W^{2} \frac{d \phi}{d z} .
\end{aligned}
$$

To make $\left(\varepsilon_{p}+\varepsilon_{k}\right)$ positive, $\frac{d W^{2}}{d z}>0$ is necessary and the profile of $W$ in Fig. $5 \mathrm{~b}$ shows that this requirement is satisfied. Near the level $U-c_{r}=0$, Eqs. (2-21) and (2-22) become

$$
\begin{aligned}
\varepsilon_{p} & =\frac{f^{2} \Lambda}{k^{3} c_{i}^{2}} W^{2} \frac{d \phi}{d z}-\frac{g \alpha S}{k c_{i}} W^{2}=\frac{W^{2}}{k c_{i}}\left[\frac{f^{2} \Lambda}{k^{2} c_{i}} \frac{d \phi}{d z}\right. \\
& -g \alpha S], \\
\varepsilon_{k} & =\frac{\Lambda W^{2}}{k} \frac{d \phi}{d z} .
\end{aligned}
$$

At this level, $\frac{d \phi}{d z}>0$ is necessary for $\left(\varepsilon_{p}+\varepsilon_{k}\right)$ to be positive, and this is satisfied though $\frac{d \phi}{d z}$ is small there (see Fig. 5a). We can see in Fig. 6 the extent to which the geostrophic and hydrostatic balances are attained in the unstable wave. A deviation from the hydrostatic balance is seen in temperature. The hydrostatic temperature $\theta_{H}$ is calculated from the obtained $p$ with the use of the relation $g_{\alpha} \theta_{H}=\frac{\partial p}{\partial z}$. If the geostrophic balance is attained, the meridional velocity $v_{G}$ is related to $p$ as $f v_{G}=\frac{\partial p}{\partial x}$, and $v_{G}$ in Fig. 6 is obtained by use of the above relation from the
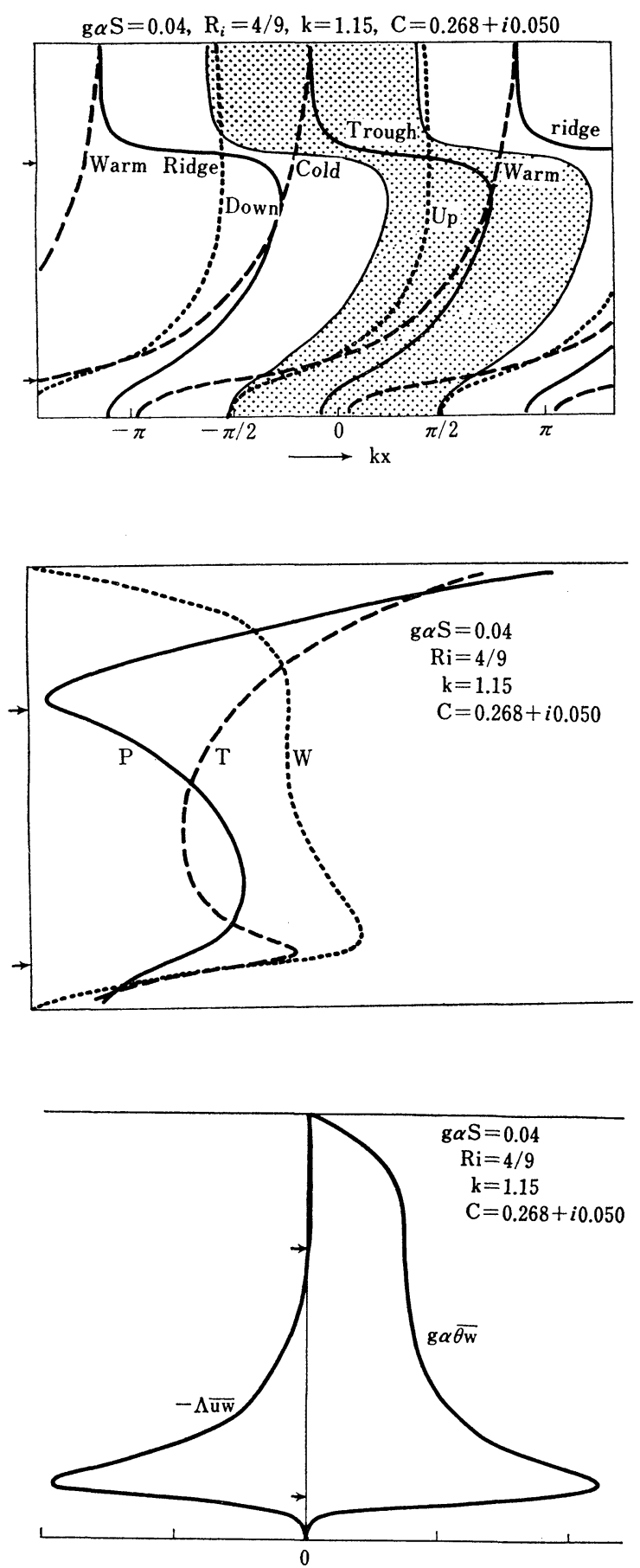

Fig. 5 A new unstable mode when $l=\beta=0$.

a) $x-z$ section of phase variation. Low pressure area is hatched.

b) Vertical distribution of amplitudes of pressure (solid line), temperature (dashed line) and vertical velocity (dotted line).

c) Energy conversions as function of $z$. 
$\mathrm{g} \alpha \mathrm{S}=0.04, \mathrm{f}=1.0, \mathrm{Z}_{0}=5.0, \mathrm{R}_{i}=4 / 9, \mathrm{k}=1.55$

$\mathrm{C}=0.389+i 0.038$
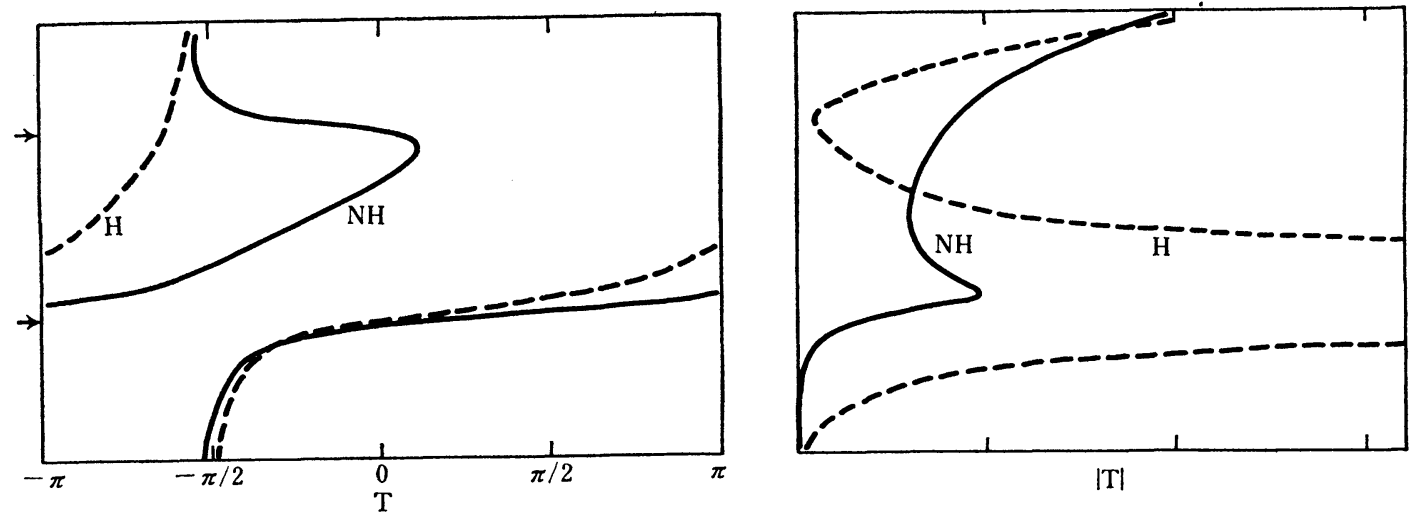

$|\mathrm{T}|$
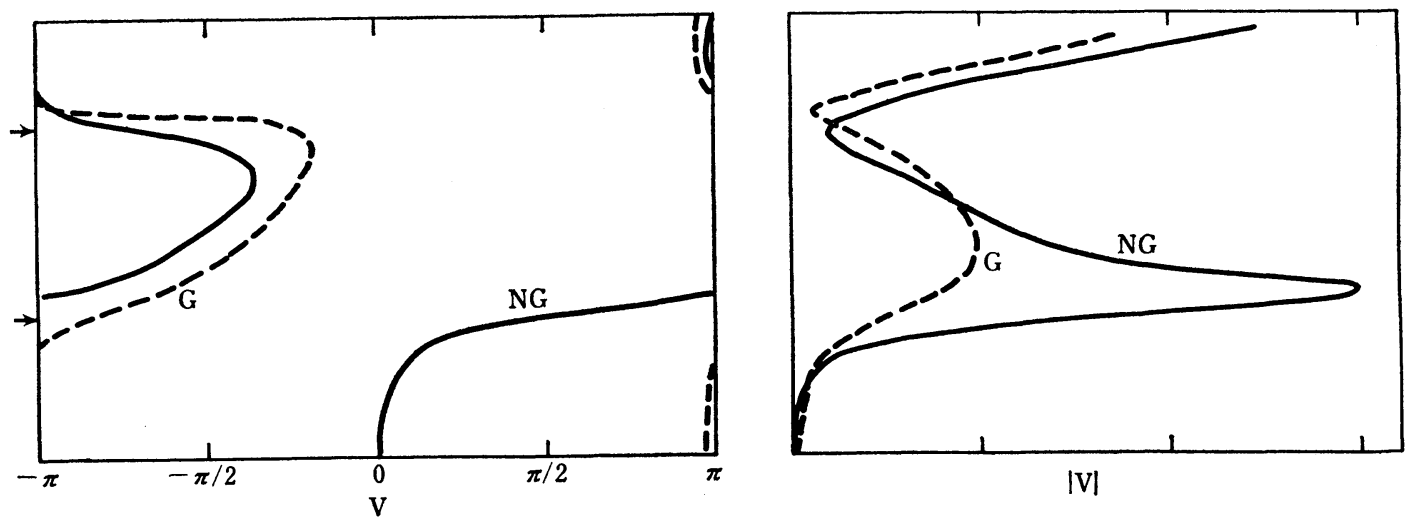

|V|

Fig. 6 The degree of non-geostrophic and non-hydrostatic deviations in the new unstable mode when $l=\beta=0$. Upper figures denote the phase (left) and the amplitude (right) of temperature. $N H$ denotes the calculated temperature, and $H$ the hydrostatic balanced temperature to the calculated pressure. Lower figures show the phase and the amplitude of meridional velocity. $N G$ denotes the calculated values and $G$ the geostrophically balanced ones to the calculated pressure.

calculated $p$. It is noted that except the region near the upper boundary, the geostrophic and hydrostatic balances do not hold for this mode. It should be noted, however, that this mode exists under the hydrostatic condition, though Fig. 6 shows some influence of the non-hydrostatic condition on this mode. This is because the nonhydrostatic condition does not change the characteristics of the singularities.

\subsection{The effect of the non-hydrostatic condition}

Orlanski (1968) and Stone (1966) showed the existence of Helmholtz type instability in the frontal model and in the continuous model respectively. Stone (1966) showed the existence of the instability when $R i<1 / 4$. In the present case, however, the Helmholtz type wave does not exist. In the following, we study the reason for this difference.

Helmholtz type waves aquire their energy through the vertical shear in the stably stratified fluid, and the waves are characterized with the smallness of latitudinal velocity. The Coriolis effect does not play a dominant role for this wave. Then after setting $v=f=0$ in Eqs. (2-9) to (2-13), we obtain

$$
\frac{d^{2} w}{d z^{2}}-k^{2}\left[1-\frac{g \alpha S}{k^{2}(U-c)^{2}}\right] w=0,
$$

corresponding to Eq. (2-20). If we change the independent variable $z$ to $\eta=k(U-c) / \Lambda$,

$$
\frac{d^{2} w}{d \eta^{2}}-\left(1-\frac{R i}{\eta^{2}}\right) w=0,
$$

is obtained. The characteristics of the above equation was discussed by Kuo (1963) precisely, which indicates that unless $R i<-3 / 4$, no unstable 
mode which makes an exponential growth exists.

If we further assume the hydrostatic balance, the governing equation becomes

$$
\frac{d^{2} w}{d \eta^{2}}+\frac{R i}{\eta^{2}} w=0 .
$$

The solution of $(5-6)$ is given by

$$
w=\alpha_{1} \eta \varepsilon_{1}+\alpha_{1} \eta \varepsilon^{\varepsilon_{2}},
$$

where $\alpha_{1}$ and $\alpha_{2}$ are constants, and

$$
\left(\begin{array}{l}
\varepsilon_{1} \\
\varepsilon_{2}
\end{array}\right)=\frac{1}{2}(1 \pm \sqrt{1-4 R i}) .
$$

The characteristic equation is written as

$$
e^{i(\phi-\psi)\left(\varepsilon_{1}-\varepsilon_{2}\right)}=\left(\frac{F}{E}\right)^{\left(\varepsilon_{1}-\varepsilon_{2}\right)}
$$

where

$$
\eta\left(z_{0} / 2\right)=E e^{i \phi} \quad \text { and } \quad \eta\left(-z_{0} / 2\right)=F e^{i \psi}
$$

$\phi, \phi$ : real; $E, F$ : real and positive.

When $R i<1 / 4$ (or $\varepsilon_{1}$ and $\varepsilon_{2}$ are real), the right hand side of the characteristic equation becomes real. Then the following relations are required;

$$
(\phi-\phi)\left(\varepsilon_{1}-\varepsilon_{2}\right)=2 m \pi \quad(m=0, \pm 1, \cdots)
$$

and

$$
\frac{E}{F}=1 \text {. }
$$

If we rearrange the above relations, we get the following results;

$$
\begin{aligned}
& c_{r}=0, \\
& \phi \sqrt{1-4 R i}=m \frac{\pi}{2}, \quad\left(\phi=\tan ^{-1} \frac{2 c_{i}}{\Lambda z_{0}}\right) .
\end{aligned}
$$

On the other hand, when $R i>1 / 4, \varepsilon_{1}-\varepsilon_{2}(=i \delta)$ becomes pure imaginary ( $\delta$ : real). The characteristic relation becomes

$$
e^{-(\phi-\psi) \delta}=\left(\frac{F}{E}\right)^{i \delta} \text { or }-(\phi-\psi) \delta=i \delta \ln \frac{F}{E} .
$$

The left hand side of the above equation is real. As $\delta$ is not equal to zero, $\phi-\phi=0$ and $E=F$ are required. This suggests that there exists no normal mode solution for $R i>1 / 4$.

From the above discussions, it is concluded that the hydrostatic assumption has a great influence on the Helmholtz type wave, i.e., this wave becomes stable in the range of positive $R i$ when we do not make the hydrostatic assumption. This may be because the hydrostatic balance cuts off the direct relation between the vertical acceler- ation and the pressure field.

\section{Non-geostrophic and non-hydrostatic solutions when $l \neq 0, \beta=0$}

\subsection{Phase velocity and growth rate}

The results of the numerical calculations for the case of $l \neq 0$ and $\beta=0$ is presented in Fig. 7 . Physical parameters are chosen as follows; $g_{\alpha} S=$ $0.04 \mathrm{sec}^{-2}, z_{0}=5.0 \mathrm{~cm}, f=1.0 \mathrm{sec}^{-1}, \quad R i=4 / 9$ $\left(\Lambda=0.3 \mathrm{sec}^{-1}\right) . c_{r}$ and $c_{i}$ for negative $l$ are also calculated but are omitted in Fig. 7 as the numerical values are nearly symmetric with respect to the $l=0$ axis. The value of $c$ is slightly larger in the positive $l$ domain than in the negative $l$ domain. The difference is $1 \%$ at the greatest in the wave number space calculated.

There is a growth rate peak on the $l=0$ axis. This is the ordinary baroclinic mode obtained by Eady (1949). There are other growth rate peaks at large $|l|$ when $k \rightarrow 0$, as suggested by Stone (1966). The latter peaks give greater growth rates than the peak due to the baroclinic mode. Stone (1966) concluded that the symmetric instability gives the greater growth rate than that of the baroclinic instability when $R i$ is less than 0.95 ,

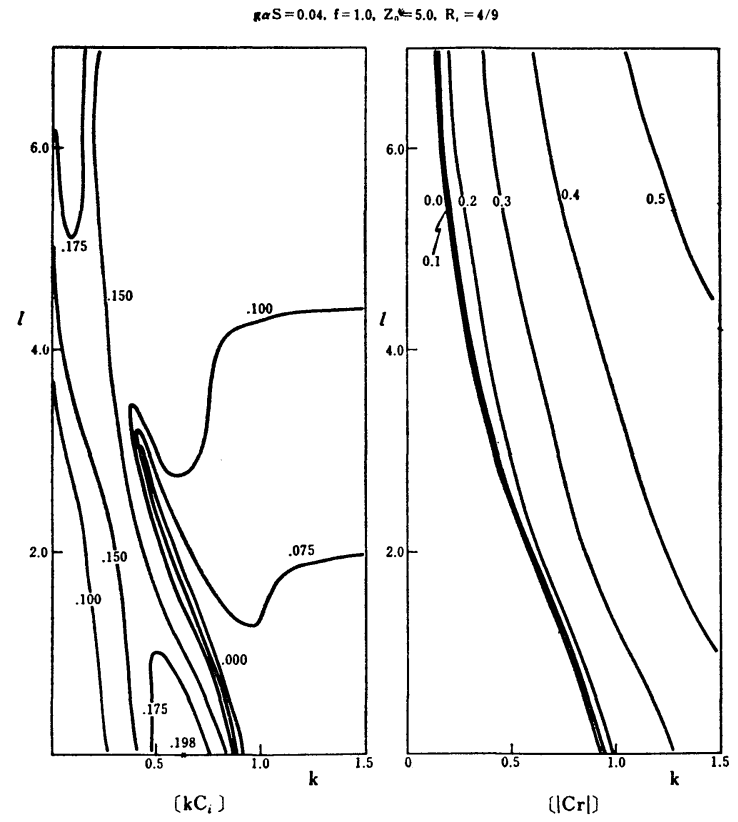

Fig. 7 The growth rate (left) and the phase velocity (right) when $\beta=0$. The ordinate is the latitudinal wave number and the abscissa the longitudinal wave number. (units in c.g.s.) 
and the present calculation confirms his result. When $k=0$, the symmetric instability appears in the range

$$
l^{2}>\frac{1}{1-R i}\left(\frac{\pi f}{\Lambda z_{0}}\right)^{2}
$$

(cf. Stone (1966)). This critical value of $l$ is about 2.8 in the present case. There is' a saddle point of growth rate near $k \sim 0.3$ and $l \sim 2.7$. The growth rate peak for a moderate $l$ and small $k$ suggested by Gambo (1970b) did not appear in the present non-geostrophic case.

Fig. 8 shows the phase velocity and growth rate curves at $l=8$. Two modes of symmetric instability coincide at $k=0.106$. From that point non-zero phase velocities appear, and this indicates the existence of two modes $c_{r}+i c_{i}$ and $-c_{r}+i c_{i}$ from the symmetry considerations. When we see Figs. 7 and 8 , the new mode at $l=0$ discussed

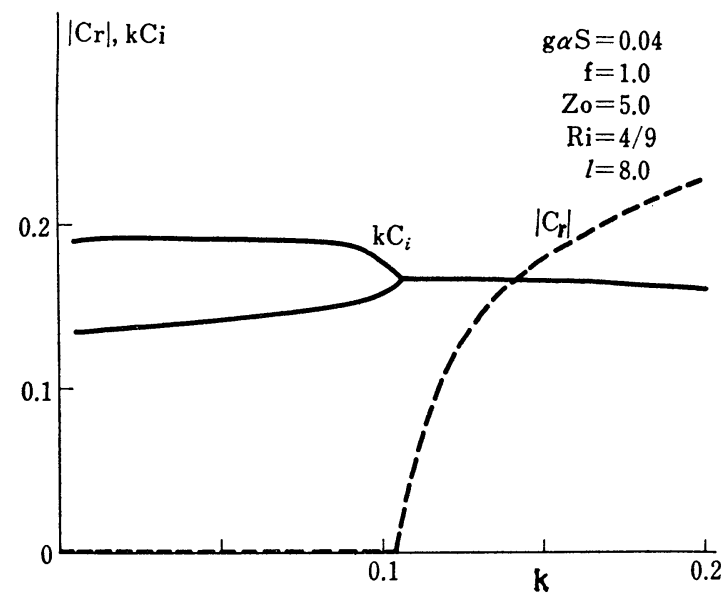

Fig. 8 The growth rate (solid line) and the phase velocity (dashed line) at $R i=8$ when $\beta=0$. (units in $c . g . s$. ) in the previous section is understood as an inertial mode continued from the symmetric instability.

\subsection{Structure and energetics of the unstable modes}

Characteristic features of the Eady's mode at a small $l$ which is obtained under the non-geostrophic and non-hydrostatic conditions are shown in Fig. 9. The trough line inclines westward and the phase of temperature inclines eastward. Amplitudes of pressure and temperature are minimum at the mid level. These features differ little from those under the geostrophic approximation.

We shall compare the structures of unstable waves of positive and negative latitudinal wave numbers at $k=0.1$ in Fig. 10. As a whole, in the positive region of $l$, phase lines of the disturbances incline eastward, while they incline westward when $l$ is negative. Figs. $10 \mathrm{a}$ and $10 \mathrm{~b}$ are representatives of the region where $n^{2}=R i-$ $(l / k)^{2}(1-R i)$ is negative and $l^{2}-\frac{R i}{1-R i} k^{2}<\frac{1}{1-R i}$ $\times\left(\frac{\pi f}{\Lambda z_{0}}\right)^{2}$. Contrary to the Eady's mode presented in Fig. 9, the amplitude of the vertical velocity is remarkably large. It is also noted that the phase of temperature strongly inclines eastward in the case $l=2.7$. Figs. $10 \mathrm{c}$ and $10 \mathrm{~d}$ show the structure of waves of $l=6.0$ and -6.0 respectively. A sharp increase of the amplitude of $w$ is remarkable. More interesting is a change of mutual phase relations among pressure, temperature and vertical velocity from the cases of $l= \pm 2.7$ to those of $l= \pm 6.0$. In the mid-level, the phases of maximum temperature and vertical velocity lag behind the trough line by $\pi / 2$ in the cases of $l=6.0$ and -6.0 , while Figs. $10 \mathrm{a}$ and $10 \mathrm{~b}$ show that the phases of maximum temperature and
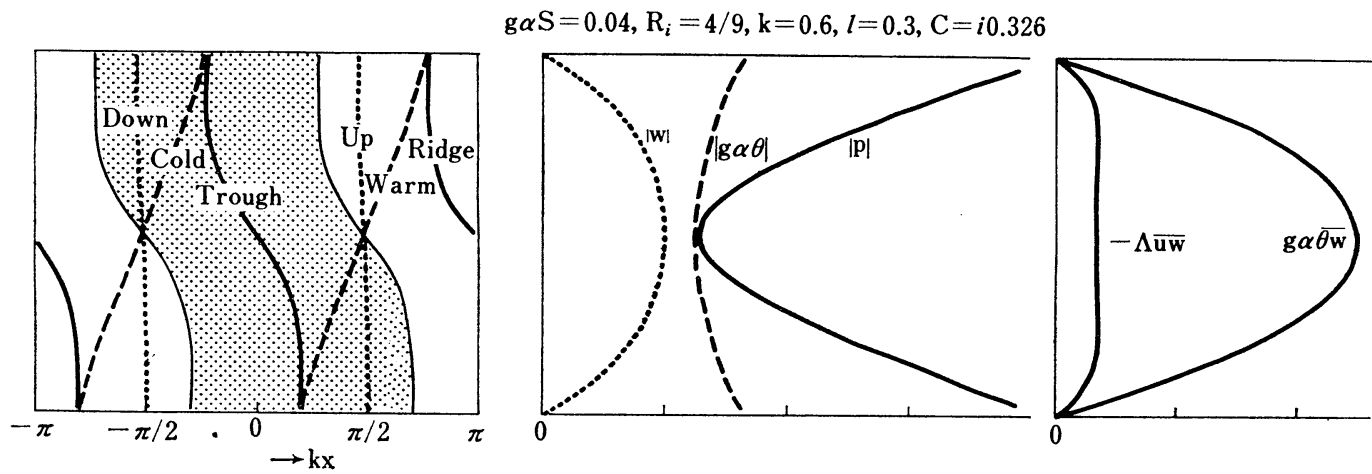

Fig. 9 Vertical distributions of the phase (left), the amplitude (center) and energy conversions (right) when $\beta=0, k=0.6$ and $l=0.3$. 

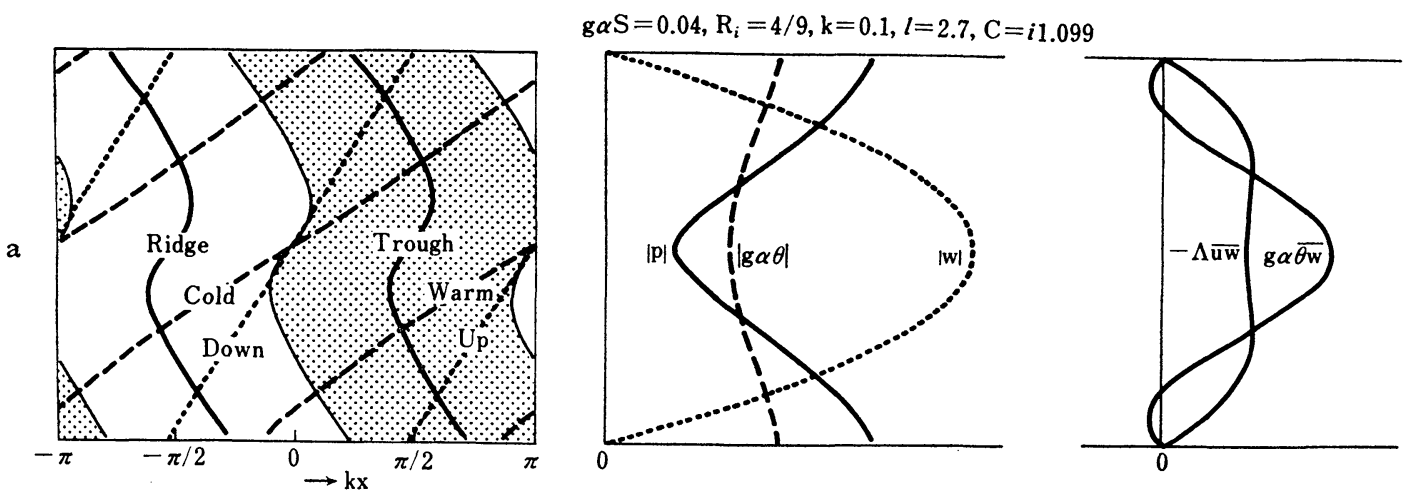

b
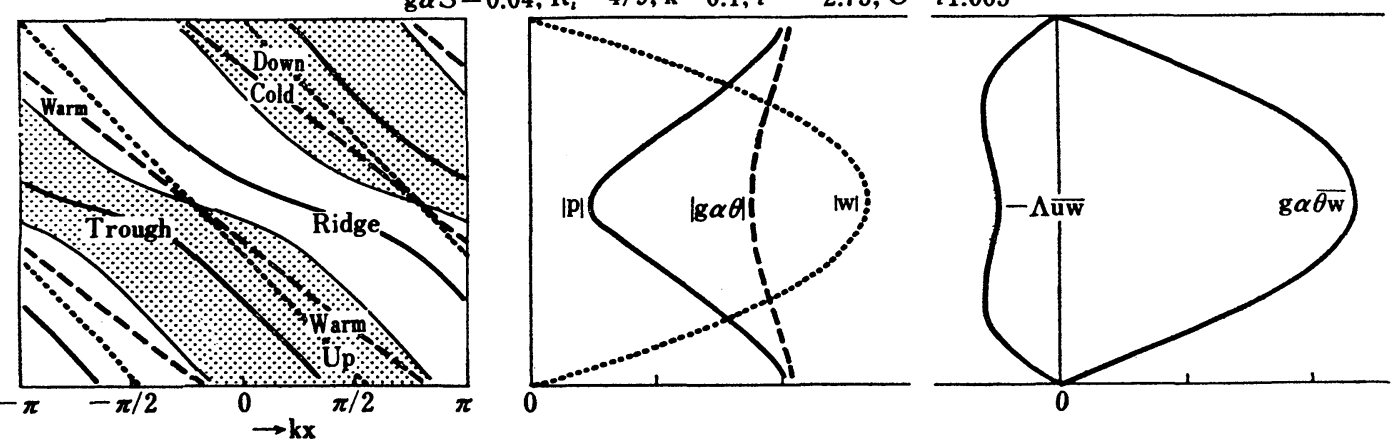

$\mathrm{g} \alpha \mathrm{S}=0.04, \mathrm{R}_{i}=4 / 9, \mathrm{k}=0.1, l=6.0, \mathrm{C}=i 1.801$
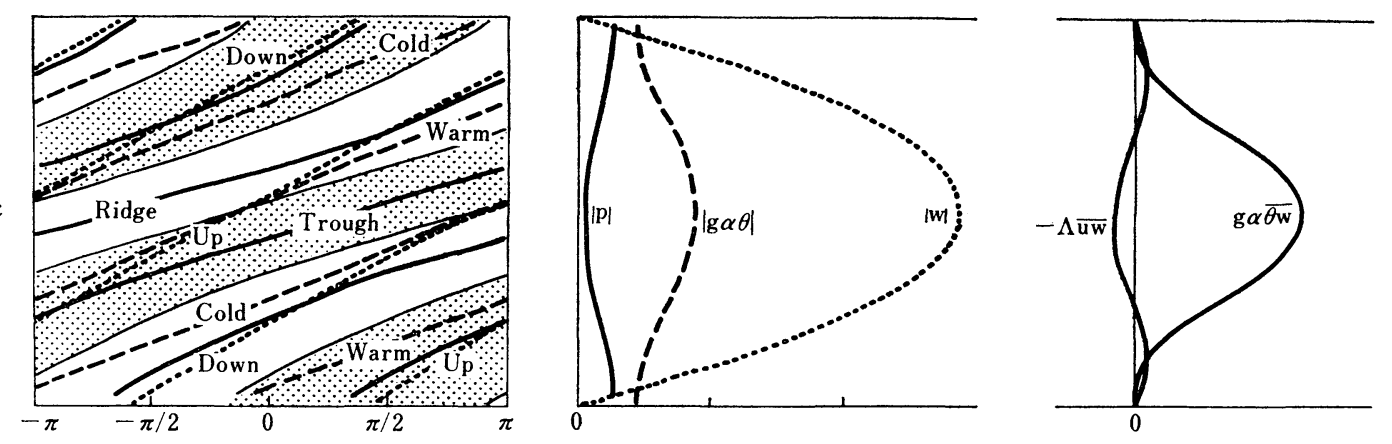

$\mathrm{g} \alpha \mathrm{S}=0.04, \mathrm{R}_{i}=4 / 9, \mathrm{k}=0.1, l=-6.0, \mathrm{C}=i 1.773$
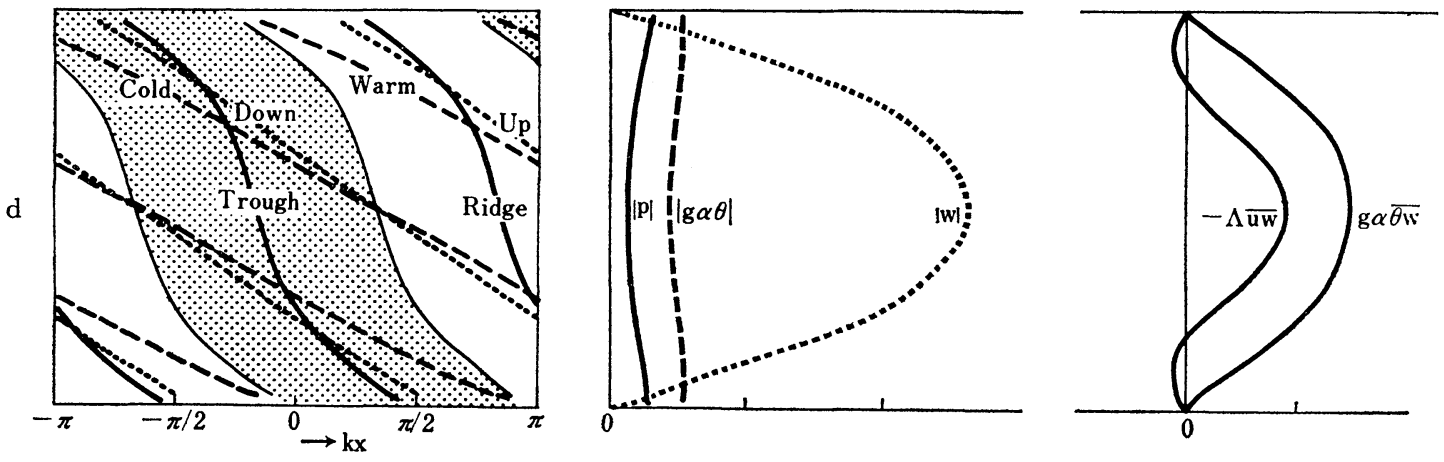

Fig. 10 Same as Fig. 9 but for $k=0.1$ and $l=2.7,-2.75, \pm 6.0$. 
upward velocity precede the trough line by $\pi / 2$. This phase change occurs at $|l|=4.2$ when $k=$ 0.1 .

We shall consider the reason for this abrupt change of the phase relations. Perturbation pressure $p$ and temperature $\theta$ are related to the vertical velocity $w\left(=W e^{i \phi}\right)$ through Eqs. (2-9) to (2-13) as follows at the mid-level, when $U(0)-$ $c_{r}=0$,

$$
\begin{aligned}
p=i A w, \quad A & =\frac{1}{M k c_{i}}\left[\left(\Lambda c_{i}-\phi^{\prime} c_{i}^{2}\right) k^{2}\right. \\
& \left.-f\left(l \Lambda+f \phi^{\prime}\right)\right], \\
\theta=D w, \quad D & =\frac{1}{M k c_{i}}\left[\frac{f l \Lambda^{2}}{c_{i}}-g \alpha S M\right. \\
& \left.+\frac{f \Lambda\left(f-l c_{i}\right)}{c_{i}} \phi^{\prime}\right] .
\end{aligned}
$$

In deriving these relations, $d W / d z=0$ at the midlevel is used, which is plausible when we see the symmetry of the governing equation with respect to $z=0$. $A$ and $D$ are real functions, then

$$
\begin{aligned}
& \arg p-\arg w=\pi / 2 \text { or }-\pi / 2, \\
& \arg \theta-\arg w=0 \text { or } \pi,
\end{aligned}
$$

is required for this mode. If $k$ approaches zero, $A$ is approximated as

$$
A \sim-\frac{f}{l^{2} k c_{i}}\left(l \Lambda+f \phi^{\prime}\right) .
$$

Then the sign $\left(l \Lambda+f \phi^{\prime}\right)$ determines the phase relation between $p$ and $w$ as follows,

$$
\arg p=\arg w \mp \pi / 2 \text { when } l \Lambda+f \phi^{\prime} \gtrless 0 .
$$

In the limit $|l| \rightarrow \infty, c_{i} \rightarrow \infty,\left|\phi^{\prime}\right| \rightarrow \infty, D$ is approximated as

$$
D \sim-\frac{1}{k c_{i}}\left[g_{\alpha} S+\frac{f \Lambda}{l} \phi^{\prime}\right] .
$$

Then

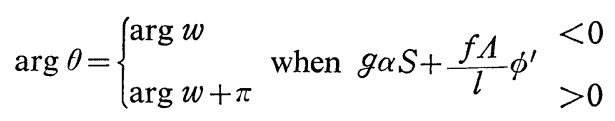

Referring to Fig. 10 and Eq. (6-6), we may discuss the followings. For a positive $l$, eastward inclination $-\phi^{\prime}$ increases in greater degree than the increase of $l$, then $g \alpha S+\frac{f A}{l} \phi^{\prime}$ is always negative $(\arg \theta=\arg w)$, while for a negative $l$ westward inclination increases in lesser degree than the increase of $-l$, then $g_{\alpha} S+\frac{f A}{l} \phi^{\prime}$ becomes positive for a certain negative $l(\arg w+\pi=\arg \theta)$. Thus, $\theta w$ correlation for a large negative $l$ becomes negative near the mid-level (see Fig. 11). Therefore the energy supply from the potential energy is dominant for the positive $l$ mode, while the energy input from the basic kinetic energy through the vertical shear is relatively large for the negative $l$ mode. The great difference of the structure

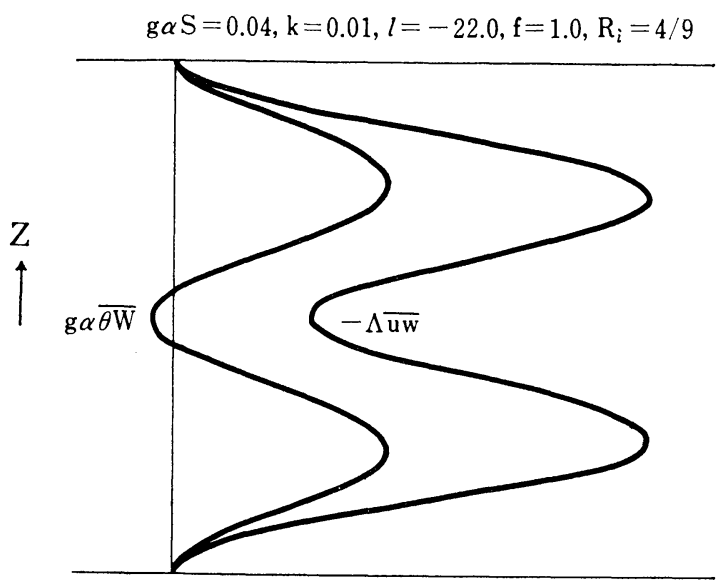

Fig. 11 Vertical distributions of energy conversion terms when $\beta=0, k=0.01$ and $l=-22.0$.
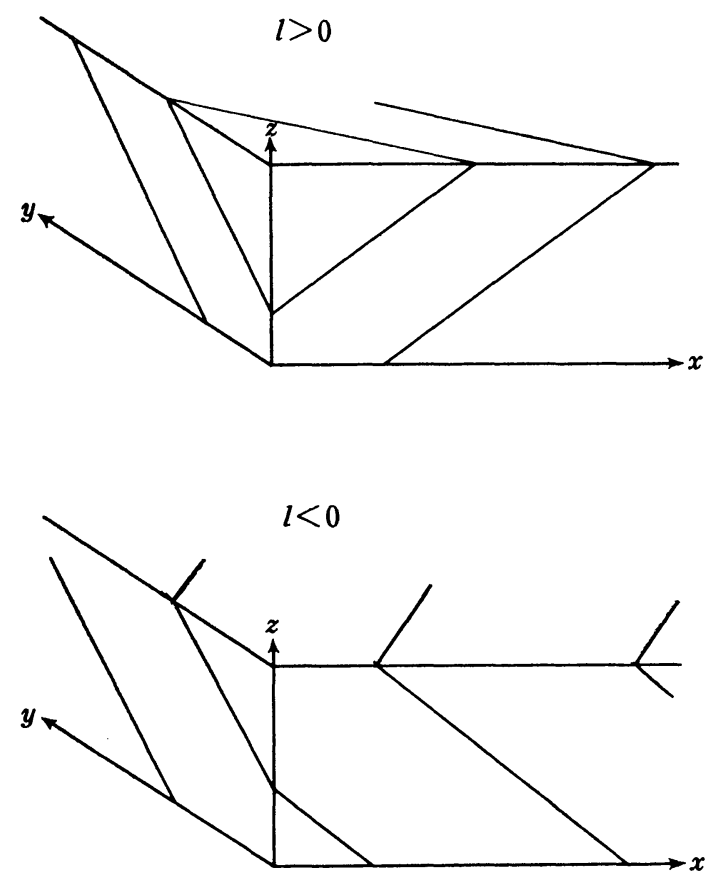

Fig. 12 Schematical structure of waves with positive and negative $l$ when $|l|$ is large and $k$ is small. 
of waves of positive and negative $l$ in the $x-z$ section suggests that the characteristics of the waves are close to those of a symmetric instability, and that the motions are more controled in the meridional plane than in the zonal plane. Fig. 12 shows a schematical view of phase lines in the zonal and the meridional sections for positive and negative $l$. In the meridional section, phase inclination is the same sense for both signs of $l$, contrary to the differences in the zonal section. It is seen from Figs. $10 \mathrm{c}$ and $10 \mathrm{~d}$ that the inclination angle tends to $\vartheta=\pi-\tan ^{-1}(B / S)$ with the increase of $|l|$.

Yanai and Tokioka (1969) made a numerical experiment of a symmetric instability in several cases, among which is a pure baroclinic case where the perturbation energy was supplied from the basic kinetic energy and the potential energy was stored up. This is not a surprise as noted in that paper when we examine energy conversion terms. This situation can be explained with the knowledge of the present study, i.e., the symmetric instability is understood as a limiting case of $k \rightarrow 0$, then the potential energy becomes the source of the meridional motion for a large positive $l$, while the basic kinetic energy plays an important role for a large negative $l$.

Fig. 13a shows the structure of an unstable wave when $c_{r}$ is not equal to zero and $l$ is large. The main features differ little from those of $c_{r}=0$ (see Fig. 10c). The structural change from $c_{r}=0$ to $c_{r} \neq 0$ is gradual and continuous. Fig. $13 \mathrm{~b}$ is an unstable mode at $k=0.2$ and $l=2.75$. The structure is very similar to that of the new mode reported in the previous section (see Fig. 5). From the structural stand point, the continuation of a symmetric instability to the unstable mode at a large $k$ and $l=0$ is apparent.

\section{Notes on the model including the $\beta$-effect}

'Arnason (1963) showed the existence of short wave cutting under the non-geostrophic condition even when there is the $\beta$-effect, contrary to the results of Green (1960) and Burger (1962) which were obtained under the geostrophic assumption. 'Arnason derived the above result by use of the
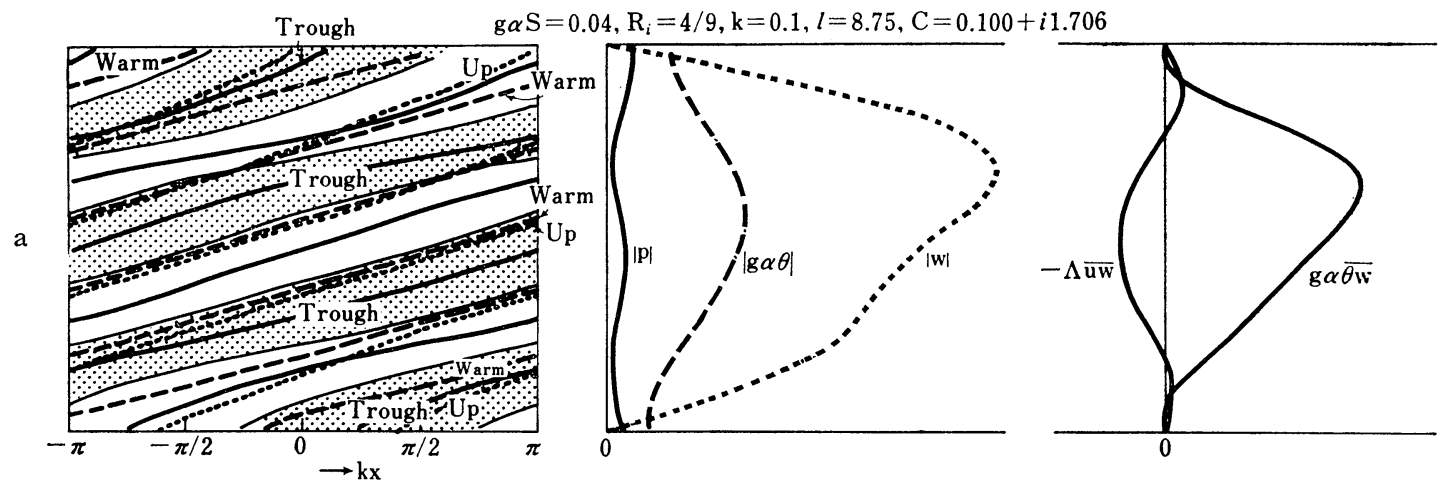

$\mathrm{g} \alpha \mathrm{S}=0.04, \mathrm{R}_{i}=4 / 9, \mathrm{k}=0.50, l=2.75, \mathrm{C}=0.212+i 0.147$

b

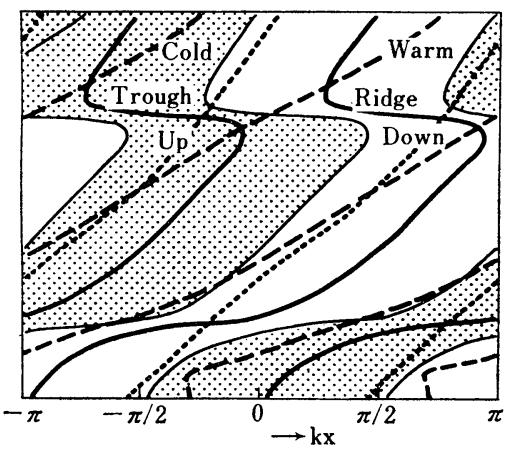

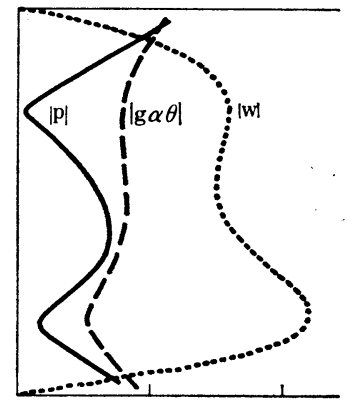

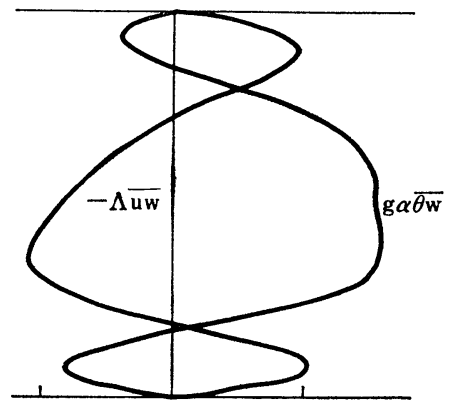

Fig. 13 Same as Fig. 9 but for $k=0.1, l=8.75$ and $k=0.5, l=2.75$. 
power series expansion around the level where $U-c_{r}=0$.

We also treat this problem under non-geostrophic and non-hydrostatic conditions. Eq. (2-20) is solved by use of the same iterative method as stated before. Fig. 14 and Fig. 15 show the results, where $g \alpha S=0.004 \mathrm{~cm}^{-2}, f=1.0 \mathrm{sec}^{-1}, R i$ $=0.4, \beta=0.02 \mathrm{sec}^{-1} \mathrm{~cm}^{-1}$. In the transitional zone from the ordinary baroclinic mode to the inertial mode at large $k$, the convergence of the iteration was not attained. In the inertial regime, however, there are two unstable modes and their structures differ little from those obtained when $\beta=0$.

The second baroclinic mode in the long wave side (see Green (1960) or Hirota (1968) for example) did not appear in the present calculations.

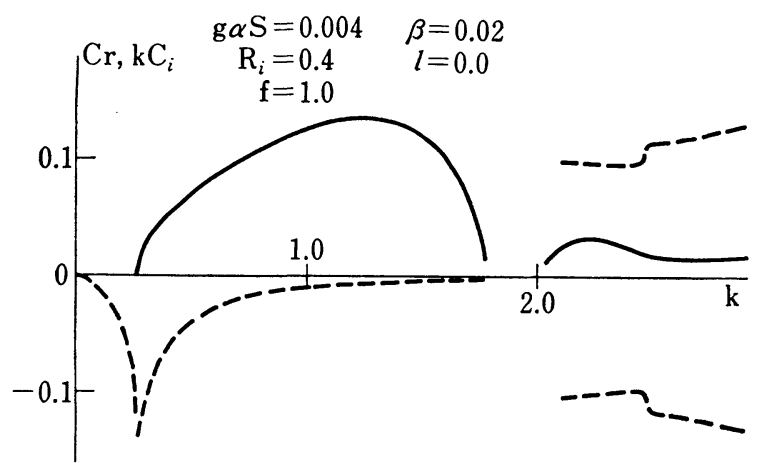

Fig. 14 The growth rate (solid line) and the phase velocity (dashed line) when $R i=0.4, \beta=$ $0.02 \mathrm{~cm}^{-1} \mathrm{sec}^{-1}, l=0$. (units in $c . g . s$.)

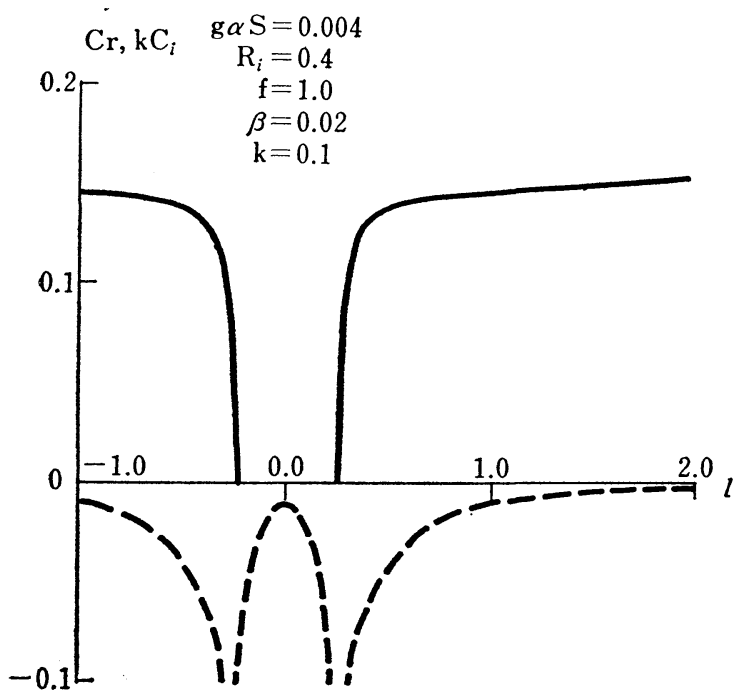

Fig. 15 Same as Fig. 14 but for $k=0.1$.
If we set $l=0$ in Eq. $(2-20)^{\prime}$, the following equation results;

$$
\begin{aligned}
& Y[Y(Y-2 a)-1] \frac{d^{2} w}{d Y^{2}}+\frac{1}{F}\left\{F+\frac{M}{k} Y\right. \\
& \left.+a \frac{M}{k} Y^{2}\right\} \frac{d w}{d Y}+\frac{f^{2} F}{k \Lambda^{2}}\left\{\frac{g \alpha S}{f^{2}}-Y^{2}\right\} w=0
\end{aligned}
$$

Near the level where $Y \sim 0$, we obtain the following equation,

$$
Y \frac{d^{2} w}{d Y^{2}}-\frac{d w}{d Y}+a R i w=0
$$

This form of the equation assures that $Y=0$ is a real singular point. The neutral mode in Fig. 14 and Fig. 15 contains the level where $Y=0$ in the layer. This is a contradiction to the concept of critical layer instability. When we choose physical parameters as $g \alpha S=0.04 \mathrm{sec}^{-2}, f=1.0$ $\mathrm{sec}^{-1}, \quad R i=4.0, \quad \beta=0.2 \mathrm{sec}^{-1} \mathrm{~cm}^{-1}$, there appears the second barclinic mode (see Fig. 16 and Fig. 17). The growth rate of the second mode is very small compared with the usual baroclinic mode. As no mathematical differences exists in the behaviour of the differential equation between the above two cases of parameters, the reason for the disappearance of the second mode in the former case may be due to a truncation error because of poor resolution in the numerical calculation. Eq. (7-1) is written in $z$-coordinate as follows;

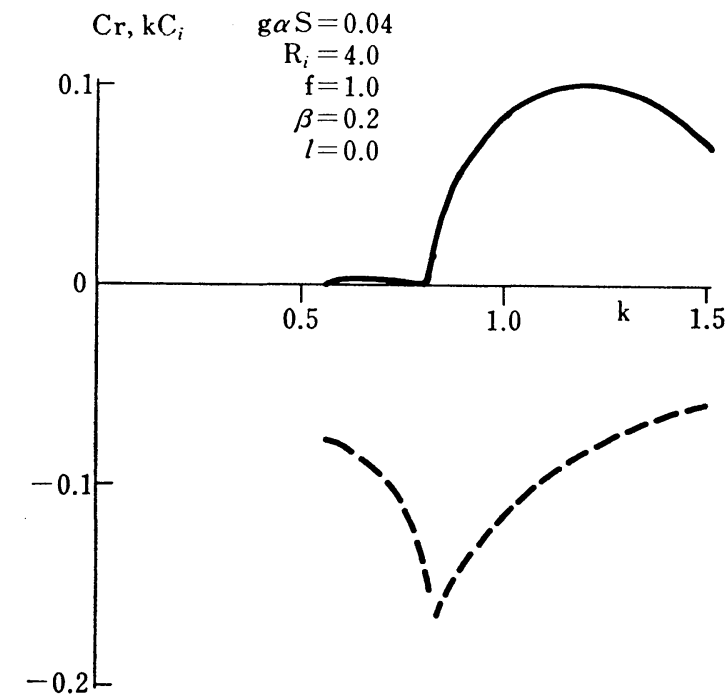

Fig. 16 The growth rate (solid line) and the phase velocity (dashed line) when $R i=4.0, \beta=$ $0.2 \mathrm{~cm}^{-1} \mathrm{sec}^{-1}, l=0$. (units in c.g.s.) 


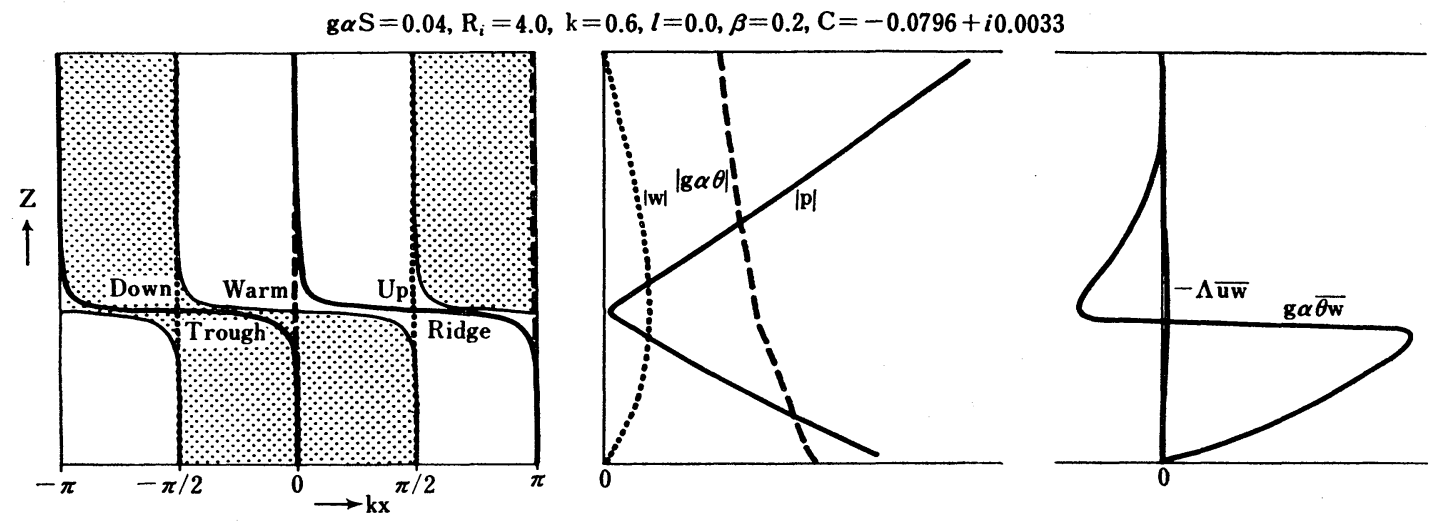

Fig. 17 Same as Fig. 9 but for $\beta=0.2 \mathrm{~cm}^{-1} \mathrm{sec}^{-1}, k=0.6, l=0$.

$$
(U-c) \frac{d^{2} w}{d z^{2}}-\Lambda \frac{d w}{d z}+\frac{g \alpha S \beta}{f^{2}} w=0 .
$$

In the former case, $g_{\alpha} S \beta / f^{2}$ is extremely small. In the numerical computations, the smallness of the coefficient of $w$ may be considered as if that term is absent, and $Y=0$ is treated as a regular point. The above discussion suggests also that the growth rate of the second baroclinic mode is greatly influenced by the value $g \alpha S \beta / f^{2}$, small value of it gives a small growth rate and vice versa.

\section{Conclusions}

We studied the stability of Eady's baroclinic model relaxing the constraints of geostrophic and hydrostatic balances and including the latitudinal variation of perturbations. The followings are concluded;

(i) There are two growth rate peaks, one is that of the ordinary baroclinic mode at $l=0$ and the other is that of the symmetric instability at $k=0,|l|=\infty$ when $R i<1$, as concluded by Stone (1966) from the study of several limiting cases. A growth rate peak at an intermediate value of $l$ and small $k$ suggested by Gambo (1970b) was not obtained in the range of parameters so far examined.

(ii) There is little difference of eigenvalues between the cases of positive and negative $l$, but the structures of unstable waves differ greatly between them in the $x-z$ plane. This is because the characteristics of the wave for large $|l|$ and small $k$ when $R i<1$ is close to those of symmetric instability, and the motions are more controled in the meridional plane. For positive $l$, the energy conversion from potential energy is domi- nant, while for negative $l$, the energy conversion from the basic kinetic energy through vertical shear becomes important.

(iii) The short wave cutting of the ordinary baroclinic mode shifts to smaller $R_{o}$. 'Arnason (1963) also got the same tendency under nongeostrophic but hydrostatic conditions. When we make the hydrostatic assumption, the short wave cutting shifts to smaller $R_{o}$ but in lesser degree than that under the non-hydrostatic case. When we fix the static stability and increase the vertical shear, the most preferred scale of the baroclinic mode moves to larger scale as $R i$ decreases.

(iv) The maximum growth rate of the ordinary baroclinic mode increases as $R i$ decreases. When the static stability is decreased while the vertical shear is fixed, the most preferred scale of the baroclinic mode decreases. These agree with the criterion of Eady (1949) and Gambo (1970a).

(v) The neutral mode $p^{+}$and $p^{-}$in Eady's solutions, are destabilized by the inertial effect in conformity with the concept of critical layer instability presented by Bretherton (1966). The resulting new unstable mode grows by the energy conversion from poetntial energy when $l=0$, and is seen as a continuation from the symmetric instability, although the latter appears only when $R i<1$, while the former exists even when $R i>1$.

(vi) If we assume the hydrostatic balance, Helmholtz type instability appears when $R i<1 / 4$ as was already noted by Stone (1966), but when we use the non-hydrostatic condition, Helmholtz type instability does not occur in the range of positive $R i$.

(vii) The effect of $\beta$ was also studied. Though the convergence of iteration was not attained in the transitional zone from the ordinary baroclinic 
mode to the inertial mode, we may say that the ordinary baroclinic mode does not continuously exist in the range of large $k$ contrary to the result obtained under geostrophic assumptions (see Green (1960) for example). 'Arnason obtained the same result by the method of power series expansion around $Y=0$. The second baroclinic mode in the long wave side exists indifferent of the value of $R i$.

\section{Acknowledgements}

The author wishes to express his hearty thanks to Prof. K. Gambo and Dr. I. Hirota of Tokyo University and to Mr. M. Uryu of Kyushu University for their discussions and comments. $\mathrm{He}$ is also indebted to Prof. M. Yanai for his encouragement throughout this work and careful reading the manuscript. Thanks are extended to Miss K. Tanemura for typing. The study was partially supported by Funds for Scientific Research from the Ministry of Education.

\section{References}

'Arnason, G., 1963: The stability of non-geostrophic perturbations in a baroclinic zonal flow. Tellus, 15, 205-229.

Bretherton, F. P., 1966: Critical layer instability in baroclinic flows. Quart. J. Roy. Meteor. Soc., 92, 325-334.

Burger, A. P., 1962: On the non-existence of critical wavelengths in a continuous baroclinic stability problem. J. Atmos. Sci., 19, 30-38.
Charney, J. G., 1947: The dynamics of long waves in a baroclinic westerly current. J. Meteor., 4, 135162.

Eady, E. T., 1949: Long waves and cyclone waves. Telius, 1, 33-52.

Fjørtoft, R., 1950: Application of integral theorems in deriving criteria of stability for laminar flows and for the baroclinic circular vortex. Geofys. Publ., 17.

Gambo, K., 1970a: The characteristic feature of medium scale disturbances in the atmosphere (I). $J$. Meteor. Soc. Japan, 48, 173-184.

1970b: The characteristic feature of medium scale disturbances in the atmosphere (II). J. Meteor. Soc. Japan, 48, 315-330.

Green, J. S. A., 1960: A problem in baroclinic stability. Quart. J. Roy. Meteor. Soc., 86, 237-251.

Hirota, I., 1968: On the dynamics of long and ultralong waves in a baroclinic zonal current. J. Meteor. Soc. Japan, 46, 234-249.

Kuo, H. L., 1963: Perturbations of plane Coutte flow in stratified fluid and origin of cloud streets. Physics of Fluid, 6, 195-211.

Ooyama, K., 1966: On the stability of the baroclinic circular vortex: a sufficient criterion for instability. J. Atmos. Sci., 23, 43-53.

Orlanski, I., 1968: Instability of frontal waves. $J$. Atmos. Sci., 25, 178-200.

Stone, P. H., 1966: On the non-geostrophic baroclinic stability. J. Atmos. Sci., 23, 390-400.

Yanai, M., and T. Tokioka, 1969: Axially symmetric meridional motions in the baroclinic circular vortex: a numerical experiment. J. Meteor. Soc. Japan, 47, 183-197.

\title{
非地衡風・非静力近似下の傾圧モデルの不安定
}

\author{
時 岡 達 志 \\ (東京大学理学部地球物理学教室)
}

小さいRichardson 数 $(R i)$ での傾圧モデルに対する非地衡風, 非静力近似の影響を調べた，損乱に関しては, 経

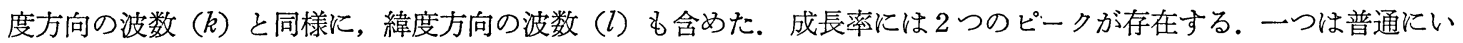
われている傾圧不安定波で, $l=0$ 上にあり, 他方は広義の慣性不安定の特殊な場合 (symmetric instability) で, $k=$ $0,|l| \rightarrow \infty$ に存在する（但し, 後者は $R i<1$ の時に限られる).この他に, 慣性の影響で不安定化した波が, 大きい 波数領域に出現する。これは，Ri<1 の時は symmetric instability とつながっている. Helmholtz type instability は現われなかつたが，これは非静力近似の影響によるものである。 $(k, l)$ と $(k,-l)$ とに対する固有值は, 殆ど異 らないが， $|l|$ が大きくなるにつれて，構造は大きくちがつてくる， $\beta$ 効果についても, 非地衡風, 非静力近似の影 響を述べる。 